\title{
Epigenetic gene silencing by heterochromatin primes fungal resistance
}

Sito Torres-Garcia, Pauline N. C. B. Audergon ${ }^{\dagger}$, Manu Shukla, Sharon A. White, Alison L. Pidoux, Robin C. Allshire*

Wellcome Centre for Cell Biology and Institute of Cell Biology, School of Biological Sciences,

The University of Edinburgh,

Mayfield Road,

Edinburgh EH9 3BF, UK.

$\dagger$ Present address: Centre for Genomic Regulation (CRG),

The Barcelona Institute of Science and Technology,

Barcelona 08003, Spain

* Corresponding author: E-mail: robin.allshire@ed.ac.uk 
1 Summary:

2 Genes embedded in H3 lysine 9 methylation (H3K9me)-dependent

3 heterochromatin are transcriptionally silenced ${ }^{1-3}$. In fission yeast,

4 Schizosaccharomyces pombe, H3K9me heterochromatin silencing can be

5 transmitted through cell division provided the counteracting demethylase Epe1

6 is absent ${ }^{4,5}$. It is possible that under certain conditions wild-type cells might

7 utilize heterochromatin heritability to form epimutations, phenotypes mediated

8 by unstable silencing rather than changes in DNA ${ }^{6,7}$. Here we show that resistant

9 heterochromatin-mediated epimutants are formed in response to threshold

10 levels of the external insult caffeine. ChIP-seq analyses of unstable resistant

11 isolates revealed new distinct heterochromatin domains, which in some cases

12 reduce the expression of underlying genes that are known to confer resistance

13 when deleted. Targeting synthetic heterochromatin at implicated loci confirmed

14 that resistance results from heterochromatin-mediated silencing. Our analyses

15 reveal that epigenetic processes allow wild-type fission yeast to adapt to non-

16 favorable environments without altering their genotype. In some isolates,

17 subsequent or co-occurring gene amplification events enhance resistance.

18 Thus, heterochromatin-dependent epimutant formation provides a bet-hedging

19 strategy that allows cells to remain genetically wild-type but transiently adapt

20 to external insults. As unstable caffeine-resistant isolates show cross-

21 resistance to the fungicide clotrimazole it is likely that related heterochromatin-

22 dependent processes contribute to anti-fungal resistance in both plant and 23 human pathogenic fungi. 
Main Text:

H3K9me heterochromatin can be copied during replication by a read-write mechanism ${ }^{4,5}$ and has been observed to arise stochastically at various loci, albeit only in the absence of key anti-silencing factors ${ }^{8-11}$. We reasoned that if heterochromatin can redistribute in wild-type $S$. pombe cells epimutations could be generated that allow cells to adapt to external insults. Unlike genetic mutants we predicted that such epimutants would be unstable, resulting in gradual loss of the resistance phenotype following growth in the absence of the external insult. To explore this possibility, we chose to test caffeine resistance because deletion of genes with a wide variety of cellular roles is known to confer resistance ${ }^{12}$, thereby increasing the chance of obtaining epimutations. We also reasoned that such unstable epimutants would occur more frequently at moderate caffeine concentrations that prevent most cells from growing $(16 \mathrm{mM})$ rather than at high stringency selection $(20 \mathrm{mM})$ used in screens for genetic caffeine-resistant mutants ${ }^{12}$.

As other secondary events might also occur upon prolonged growth on caffeine, we froze one aliquot of each isolate as soon as possible after resistant colony formation and then froze consecutive aliquots of each isolate after continued growth on caffeine (Fig. 1a). This provided a time series, permitting detection and separation of potential initiating and subsequent secondary events.

Colonies that grew after plating wild-type fission yeast $\left(972 h^{-}\right)$cells in the presence of caffeine (16 mM caffeine, +CAF) were picked. Following freezing, isolates were then successively propagated in the absence of caffeine (-CAF). Re-challenging isolates with caffeine revealed that $23 \%$ lost their caffeine resistance after 14 days of 
47 non-selective growth (denoted 'unstable isolates', UR) whereas $13 \%$ remained caffeine resistant (denoted 'stable isolates', SR). $64 \%$ of isolates did not display a clear phenotype (denoted 'unclear') (Fig. 1b, c and Extended Data Fig. 1a, b).

Deletion of $c / r 4^{+}$encoding the sole H3K9 methyltransferase in S. pombe ${ }^{13,14}$ from resistant isolates resulted in immediate loss of caffeine resistance in unstable, but not in stable isolates (Fig. 1d and Extended Data Fig. 1c), indicating that caffeine resistance in unstable isolates requires heterochromatin.

Whole genome sequencing (WGS) of the stable isolate SR-1 uncovered a mutation in pap $1^{+}$responsible for the caffeine-resistant phenotype (Extended Data Fig. 2 and ${ }^{15}$ ). ChIP-seq for H3K9me2 on SR-1 revealed no changes in heterochromatin distribution.

WGS of unstable isolates revealed no genetic changes in coding sequences involved in either caffeine resistance or H3K9me2-mediated silencing, and 8 of 30 analyzed unstable isolates had no detectable genetic change compared to wild-type

60 (Supplementary Information Table 1). ChIP-seq for H3K9me2 on unstable isolates revealed an altered heterochromatin distribution (Fig. 2a, b). Unstable resistant isolate UR-1 exhibited a new H3K9me2 domain over the hba1 locus, whereas UR-2 - UR-6 exhibited H3K9me2 domains over ncRNA.394, ppr4, grt1, fio1 and mbx2 loci, respectively (Fig. 2a, b and Supplementary Information Table 1). Deletion of $h b a 1^{+}$is known to confer caffeine resistance ${ }^{16}$, suggesting that these novel heterochromatin domains may drive caffeine resistance by silencing underlying genes. Accordingly, RT-qPCR analysis revealed reduced expression of genes underlying the observed novel heterochromatin domain at the hba1 locus (Fig. 2c). 
69 The ncRNA.394, ppr4, grt1, fio1 and mbx2 loci have not previously been implicated in caffeine resistance. Interestingly, 24 of 30 unstable isolates showed an ectopic heterochromatin domain over the ncRNA.394 locus (Extended Data Fig. 3a and Supplementary Information Table 1), and reduced levels of transcripts were present (Fig. 2c), suggesting that transcriptional silencing within this region might mediate caffeine resistance. ncRNA.394 was previously described as a heterochromatin 'island', yet H3K9me2 levels over this locus were close to background in wild-type cells and only increased in the absence of the counteracting demethylase Epe1. Our analysis failed to detect H3K9me2 over ncRNA.394 in untreated wild-type cells (Fig. $2 b$ and Extended Data Fig. 3a).

Deletion of ncRNA.394 did not result in caffeine resistance (Extended Data Fig. 3b). Prolonged non-selective growth without caffeine of cells exhibiting the ncRNA.394 $\mathrm{H} 3 \mathrm{~K} 9 \mathrm{me} 2$ domain resulted in loss of H3K9me2 over this region, whereas growth with caffeine present extended the H3K9me2 domain upstream to include the SPBC17G9.13c ${ }^{+}$and SPBC17G9.12c $c^{+}$genes (Extended Data Fig. 3c). Deletion of SPBC17G9.12c $\mathrm{C}^{+}$or eno101 ${ }^{+}$did not result in caffeine resistance (Extended Data Fig. 3b). SPBC17G9.13c is essential for viability precluding testing a deletion mutant for resistance. Together these analyses suggest that reduced expression of SPBC17G9.13c $c^{+}$may mediate caffeine resistance.

To test directly if heterochromatin formation at these specific loci can result in caffeine resistance, tetO DNA binding sites were inserted at the $h b a 1$ and $n c R N A .394$ loci and a TetR-CIr4* (catalytically active but lacking the Clr4 chromodomain) fusion protein expressed to force assembly of synthetic heterochromatin upon recruitment to these 
$92 \operatorname{loci}^{4,5}$. Combining tetO with TetR-Clr4* in the absence of anhydrotetracycline (-AHT)

93 resulted in a novel $\mathrm{H} 3 \mathrm{~K} 9 \mathrm{me} 2$ domain at each locus and growth of cells in the

94 presence of caffeine (Fig. 3 and Extended Data Fig. 4). This indicates that heterochromatin-mediated silencing at either the hba1 or ncRNA.394 loci results in caffeine resistance. Because TetR-CIr4* tethering close to SPBC17G9.13c ${ }^{+}$resulted in caffeine resistance we surmise that reduced expression of the SPBC17G9.13c ${ }^{+}$ gene upstream of $n c R N A .394$ is likely responsible for caffeine resistance at this locus.

Remarkably, we found that strains with forced synthetic heterochromatin at either hba1 or ncRNA.394 loci displayed resistance to the widely-used clinical fungicide clotrimazole (Fig. 3, +CLZ). Further investigation of our unstable caffeine-resistant isolates revealed that those with heterochromatin formation at the hba1 (UR-1) and the ncRNA.394 (UR-2) loci are also resistant to clotrimazole and generate small interfering RNAs (siRNAs) homologous to the surrounding genes (Extended Data Fig. 5).

106 In addition to a heterochromatin domain over ncRNA.394, analysis of ChIP-seq input

107 DNA indicated that many independent unstable caffeine-resistant isolates also 108 contained overlapping regions of chromosome III present at increased copy number

109 (Extended Data Fig. 6). In 11 of 12 isolates, the minimal region of overlap contains the $110 c d s 1^{+}$gene, overexpression of which is known to confer caffeine resistance ${ }^{17}$. To

111 determine if amplification of the cds1 locus occurred before or after formation of the 112 ncRNA.394 H3K9me2 domain we analyzed a sample frozen later in the time series 113 for the same isolate (UR-2). ChIP-seq analysis showed that the ncRNA.394 H3K9me2

114 domain was present in the initial caffeine-resistant isolate ( 4 days $+\mathrm{CAF}$ ), whereas the 
115 cds1 locus amplification arose later (7 days +CAF) (Extended Data Fig. 7a). These

116 data suggest that development of resistance is a multistep process in which a

117 combination of different events can increase resistance. In agreement with this

118 hypothesis, deletion of $c / r 4^{+}$in the initial UR-2 isolate (4 days $+\mathrm{CAF}$ ) resulted in loss

119 of caffeine resistance in all transformants tested (6/6) (Extended Data Fig. 7b and 1c).

120 However, only half of the transformants (3/6) lost resistance to caffeine when c/r4

121 was deleted in the isolate displaying cols1 locus amplification (7 days +CAF),

122 suggesting that once amplification of the cds1 locus occurs heterochromatin is not

123 required for resistance. In UR-2 a new heterochromatin domain occurred before

$124 \mathrm{cds} 1^{+}$amplification but it is possible that events are stochastic and occur in no fixed

125 order. Interestingly, both events - the ncRNA.394 H3K9me2 domain and cds1 locus

126 amplification - are unstable and lost following growth in the absence of caffeine

127 (Extended Data Fig. 7c).

128 To investigate the dynamics of heterochromatin domain formation in response to

129 caffeine we exposed wild-type cells to low $(7 \mathrm{mM})$ or medium $(14 \mathrm{mM})$ doses of

130 caffeine for 18 hours. Cells in low caffeine accomplished $\sim 8$ doublings, whereas fewer

131 than 3 population doublings occurred in medium caffeine. ChIP-seq for H3K9me2

132 identified several new ectopic domains of heterochromatin following exposure to low

133 caffeine. Ectopic domains were detected at loci known to accumulate H3K9me2 in

134 the absence of Epe ${ }^{8}$, including ncRNA.394 (Fig. 4a, top). Remarkably, following 135 treatment with medium doses of caffeine, ectopic heterochromatin was restricted to 136 ncRNA.394, and H3K9me2 levels at this locus were approximately 2-fold greater than

137 those after exposure to low caffeine (Fig. 4a, bottom). Together these data indicate 138 that, when exposed to near-lethal doses of caffeine (medium, $14 \mathrm{mM}$ ), wild-type cells 
140 that confers resistance when silenced.

141 To determine if other insults also induce novel heterochromatin domains, we exposed

142 wild-type cells to oxidative stress by addition of hydrogen peroxide (1 mM). ChIP-seq

143 for H3K9me2 revealed the presence of ectopic heterochromatin domains at similar

144 locations to those observed in low caffeine treatment, albeit H3K9me2 levels were

145 lower (Fig. 4b). Thus, our results reveal an adaptive epigenetic response following

146 exposure to external insults, and suggest that stress-response pathways may

147 regulate activities that modulate heterochromatin formation thereby ensuring cell survival in fluctuating environmental conditions (Extended Data Fig. 8).

149 It is well known that DNA methylation-dependent epimutations arise in plants and are

150 propagated by maintenance methyltransferases ${ }^{18,19}$. RNAi-mediated epimutations

151 have been shown to arise in the fungus Mucor circinelloides ${ }^{20}$, but it is not known if

152 these are DNA methylation or heterochromatin dependent. As fission yeast lacks DNA

153 methylation ${ }^{21,22}$ this epigenetic mark cannot be responsible for the epimutations 154 described here. Instead our analyses indicate that these adaptive epimutations are 155 transmitted in wild-type cells by the previously-identified Clr4/H3K9me read-write 156 mechanism ${ }^{4,5}$.

157 Our findings prompt the question as to why epimutants have not been detected 158 previously in mutant screens. Phenotypic screens are usually very stringent, and 159 generally only the strongest mutants are retained for further investigation and 160 eccentric mutants are discarded. Here we essentially select for weak mutants by 161 applying low doses of a drug that is at the threshold of preventing the growth of most 
cells. Selection was applied for a short period of time in order to maximize the chance

163 of identifying isolates that exhibit unstable phenotypes prior to the development of

164 genetic alterations.

165 Fungal infections are on the rise, especially in immunocompromised humans. There

166 are few effective anti-fungal agents and resistance is rendering them increasingly

167 ineffective $e^{23,24}$. The widespread use of related azole compounds to control fungal 168 deterioration of crops may leave low fungicide levels in the soil, possibly leading to

169 the unwitting selection of resistant epimutants in fungi, similar to those described

170 here, that may ultimately drive the increasing number of cases of azole-resistant

171 Aspergillosis and Cryptococcosis in the clinic. Use of the existing battery of so called

172 'epigenetic drugs' - compounds that inhibit histone modifying enzymes - may identify

173 molecules that block heterochromatin formation and hence reduce the emergence of

174 anti-fungal resistance in plant and animal pathogenic fungi. 
183 1. Bannister, A. J. et al. Selective recognition of methylated lysine 9 on histone H3 by the HP1 chromo domain. Nature 410, 120-124 (2001).

2. Lachner, M., O'Carroll, D., Rea, S., Mechtler, K. \& Jenuwein, T. Methylation of histone H3 lysine 9 creates a binding site for HP1 proteins. Nature 410, 116-

3. Allshire, R. C. \& Madhani, H. D. Ten principles of heterochromatin formation and function. Nat. Rev. Mol. Cell Biol. 45, 153 (2017).

4. Audergon, P. N. C. B. et al. Epigenetics. Restricted epigenetic inheritance of H3K9 methylation. Science 348, 132-135 (2015).

5. Ragunathan, K., Jih, G. \& Moazed, D. Epigenetics. Epigenetic inheritance uncoupled from sequence-specific recruitment. Science 348, 12586991258699 (2015).

6. Jeggo, P. A. \& Holliday, R. Azacytidine-induced reactivation of a DNA repair gene in Chinese hamster ovary cells. Mol. Cell. Biol. 6, 2944-2949 (1986).

197 7. Oey, H. \& Whitelaw, E. On the meaning of the word 'epimutation'. Trends Genet. 30, 519-520 (2014).

8. Zofall, M. et al. RNA elimination machinery targeting meiotic mRNAs promotes facultative heterochromatin formation. Science 335, 96-100 (2012). 
9. Wang, J., Reddy, B. D. \& Jia, S. Rapid epigenetic adaptation to uncontrolled heterochromatin spreading. eLife 4, 80 (2015).

10. Parsa, J.-Y., Boudoukha, S., Burke, J., Homer, C. \& Madhani, H. D. Polymerase pausing induced by sequence-specific RNA-binding protein drives heterochromatin assembly. Genes Dev. 32, 953-964 (2018).

11. Sorida, M. et al. Regulation of ectopic heterochromatin-mediated epigenetic diversification by the JmjC family protein Epe1. PLoS Genet. 15, e1008129 (2019).

12. Calvo, I. A. et al. Genome-wide screen of genes required for caffeine tolerance in fission yeast. PLoS ONE 4, e6619 (2009).

13. Ivanova, A. V., Bonaduce, M. J., Ivanov, S. V. \& Klar, A. J. The chromo and SET domains of the Clr4 protein are essential for silencing in fission yeast. Nat Genet 19, 192-195 (1998).

14. Nakayama, J., Rice, J. C., Strahl, B. D., Allis, C. D. \& Grewal, S. I. Role of histone H3 lysine 9 methylation in epigenetic control of heterochromatin assembly.

15. Kudo, N., Taoka, H., Toda, T., Yoshida, M. \& Horinouchi, S. A novel nuclear export signal sensitive to oxidative stress in the fission yeast transcription factor Pap1. J. Biol. Chem. 274, 15151-15158 (1999).

16. Castillo, E. A., Vivancos, A. P., Jones, N., Ayté, J. \& Hidalgo, E. 
a multidrug resistance phenotype due to constitutive nuclear accumulation of Pap1. J. Biol. Chem. 278, 40565-40572 (2003).

17. Wang, S. W., Norbury, C., Harris, A. L. \& Toda, T. Caffeine can override the SM checkpoint in fission yeast. J. Cell. Sci. 112 ( Pt 6), 927-937 (1999).

18. Cubas, P., Vincent, C. \& Coen, E. An epigenetic mutation responsible for natural variation in floral symmetry. Nature 401, 157-161 (1999).

19. Heard, E. \& Martienssen, R. A. Transgenerational epigenetic inheritance: myths and mechanisms. Cell 157, 95-109 (2014).

20. Calo, S. et al. Antifungal drug resistance evoked via RNAi-dependent epimutations. Nature 513, 555-558 (2014).

21. Antequera, F., Tamame, M., Villanueva, J. R. \& Santos, T. DNA methylation in the fungi. J. Biol. Chem. 259, 8033-8036 (1984).

22. Wilkinson, C. R., Bartlett, R., Nurse, P. \& Bird, A. P. The fission yeast gene pmt1+ encodes a DNA methyltransferase homologue. Nucleic Acids Res. 23, 203-210 (1995).

23. Fisher, M. C., Hawkins, N. J., Sanglard, D. \& Gurr, S. J. Worldwide emergence of resistance to antifungal drugs challenges human health and food security. Science 360, 739-742 (2018).

24. Almeida, F., Rodrigues, M. L. \& Coelho, C. The Still Underestimated Problem of Fungal Diseases Worldwide. Front Microbiol 10, 214 (2019). 


\section{Yeast strains and manipulations}

244 Standard methods were used for fission yeast growth, genetics and manipulation ${ }^{25}$.

245 S. pombe strains used in this study are described in Supplementary Information Table

246 S2. Oligonucleotide sequences are listed in Supplementary Information Table S3. For

247 pDUAL-adh21-TetR-2xFLAG-Clr4-CD $\Delta$ (abbreviated as TetR-Clr4*), the nmt81

248 promoter of pDUAL-nmt81-TetR-2xFLAG-CIr4-CD $\Delta^{4}$, was replaced by the adh21

249 promoter (pRAD21, gift from Y. Watanabe). Notl-digested plasmid was integrated at

$250 \mathrm{leu}^{+}$. Pap1-N424STOP strain and strains carrying 4xtetO insertions were

251 constructed by CRISPR/Cas9-mediated genome editing using the SpEDIT system

252 (Allshire Lab; available on request) with oligonucleotides listed in Supplementary Information Table S3. Yeast extract plus supplements (YES) was used to grow all cultures. $16 \mathrm{mM}$ caffeine (Sigma, C0750) was added to media for caffeine resistance screens and serial dilution assays. Caffeine-resistant colonies that formed after seven days were picked and patched to +CAF plates. After four days of growth, isolates were frozen ( 4 days $+\mathrm{CAF}$ ). 4 days $+\mathrm{CAF}$ isolates were repatched and grown for three days on + CAF plates and then frozen ( 7 days + CAF). Subsequently, 7 days + CAF isolates were repatched every three days on +CAF plates up to twenty days of total

260 growth on +CAF plates (20 days $+\mathrm{CAF}$ ). $0.29 \mu \mathrm{M}$ clotrimazole (Sigma, C6019) was added to media for clotrimazole resistance serial dilution assays. 7 or $14 \mathrm{mM}$ caffeine

262 (Sigma, C0750), or $1 \mathrm{mM}$ hydrogen peroxide (Sigma, H1009) were added to media

263 for 18 hours for drug treatment experiments. To release TetR-Clr4* $10 \mu \mathrm{M}$ anhydrotetracycline (AHT) was added to the media. 


\section{Serial dilution assays}

266 Equal amounts of starting cells were serially diluted four-fold and then spotted onto

267 appropriate media. Cells were grown at $30-32^{\circ} \mathrm{C}$ for $3-5$ days and then photographed.

\section{Chromatin immunoprecipitation (ChIP)}

269 ChIP experiments were performed as previously described ${ }^{26}$ using anti-H3K9me2

270 (5.1.1, a kind gift by Takeshi Urano). Immunoprecipitated DNA was recovered with

271 Chelex-100 resin (BioRad) for ChIP-qPCR (qChIP) experiments or with QIAquick

272 PCR Purification Kit (Qiagen) for ChIP-seq experiments.

\section{Quantitative ChIP (qChIP)}

274 qChIPs were analysed by real-time PCR using Lightcycler 480 SYBR Green (Roche) 275 with oligonucleotides listed in Supplementary Information Table S3. All ChIP

276 enrichments were calculated as \% DNA immunoprecipitated at the locus of interest

277 relative to the corresponding input samples and normalized to \% DNA 278 immunoprecipitated at the $a c t 1^{+}$locus. Histograms represent data averaged over

279 three biological replicates. Error bars represent standard deviations.

\section{ChIP-seq library preparation and analysis}

281 Illumina-compatible libraries were prepared as previously described ${ }^{26}$ using

282 NEXTflex-96 barcode adapters (Bioo Scientific) and Ampure XP beads (Beckman

283 Coulter). Libraries were then pooled to allow multiplexing and sequenced on an

284 Illumina HiSeq2000, NextSeq or MiniSeq system (150-cycle high output kit) by 75 bp 285 paired-end sequencing. 
Approximately 6-10 million 75 bp paired-end reads were produced for each sample.

287 Raw reads were then de-multiplexed and trimmed using Trimmomatic (v0.35) ${ }^{27}$ to

288 remove adapter contamination and regions of poor sequencing quality. Trimmed

289 reads were aligned to the $S$. pombe reference genome (972h-, ASM294v2.20) using

290 Bowtie2 (v2.3.3 $)^{28}$. Resulting bam files were processed using Samtools (v1.3.1 $)^{29}$ and

291 picard-tools (v2.1.0) (http://broadinstitute.github.io/picard) for sorting, removing

292 duplicates and indexing. Coverage bigwig files were generated by BamCoverage

293 (deepTools v2.0) and ratios IP/input were calculated using BamCompare (deepTools 294 v2.0) ${ }^{30}$ in SES mode for normalisation ${ }^{31}$. Peaks were called using MACS2 ${ }^{32}$ in PE mode 295 and broad peak calling (broad-cutoff $=0.05$ ). Region-specific H3K9me2 enrichment 296 plots were generated using the Sushi R package $(\mathrm{v} 1.22)^{33}$.

\section{SNP and indel calling}

298 SNPs and indels were called as described ${ }^{34}$. Trimmed reads were mapped to the S.

299 pombe reference genome (972h-, ASM294v2.20) using Bowtie2 (v2.3.3) ${ }^{28}$. GATK ${ }^{35,36}$

300 was used for base quality score recalibration. SNPs and indels were called with GATK

301 HaplotypeCaller ${ }^{35,36}$ and filtered using custom parameters. Functional effect of

302 variants was determined using Variant Effect Predictor ${ }^{37}$.

\section{Copy number variation analysis}

304 Copy number variation was determined using CNVkit ${ }^{38}$ in Whole-Genome 305 Sequencing (-wgs) mode. Wild-type ChIP-seq input bam files were used as reference. 
309 For qRT-PCR, total RNA was extracted using the Monarch Total RNA Miniprep Kit

310 (New England Biolabs) according to the manufacturer's instructions. Contaminating

311 DNA was removed by treating with Turbo DNase (Invitrogen) and reverse transcription

312 was performed using LunaScript RT Supermix Kit (New England Biolabs).

313 Oligonucleotides used for qRT-PCR are listed in Supplementary Information Table

314 S3. qRT-PCR histograms represent three biological replicates; error bars correspond

315 to the standard deviation. ${ }^{*} P<0.05$ ( $t$ test).

316 Small RNA-seq

$31750 \mathrm{~mL}$ of log-phase cells were collected and processed using the mirVana miRNA

318 Isolation kit (Invitrogen). Resulting sRNA was treated with TURBO DNase

319 (Invitrogen) and used for library construction using NEBNext Multiplex Small RNA

320 Library Prep Set for Illumina (New England Biolabs) according to manufacturer's

321 instructions. Libraries were pooled and sequenced on an Illumina NextSeq platform

322 by 50 bp single-end sequencing. Raw reads were then de-multiplexed and

323 processed using Cutadapt (v1.17) to remove adapter contamination and discard

324 reads shorter than 19 nucleotides or longer than 25 nucleotides. Coverage plots were

325 generated using SCRAM ${ }^{39}$. 
bioRxiv preprint doi: https://doi.org/10.1101/808055; this version posted October 17,2019 . The copyright holder for this preprint (which

was not certified by peer review) is the author/funder. All rights reserved. No reuse allowed without permission. 
25. Moreno, S., Klar, A. \& Nurse, P. Molecular genetic analysis of fission yeast

26. Tong, P. et al. Interspecies conservation of organisation and function between nonhomologous regional centromeres. Nat Commun 10, 2343 (2019).

27. Bolger, A. M., Lohse, M. \& Usadel, B. Trimmomatic: a flexible trimmer for Illumina sequence data. Bioinformatics 30, 2114-2120 (2014).

28. Langmead, B. \& Salzberg, S. L. Fast gapped-read alignment with Bowtie 2. Nat. Methods 9, 357-359 (2012).

29. Li, H. et al. The Sequence Alignment/Map format and SAMtools. Bioinformatics 25, 2078-2079 (2009).

30. Ramírez, F. et al. deepTools2: a next generation web server for deepsequencing data analysis. Nucleic Acids Res. 44, W160-5 (2016).

31. Diaz, A., Park, K., Lim, D. A. \& Song, J. S. Normalization, bias correction, and peak calling for ChIP-seq. Stat Appl Genet Mol Biol 11, Article 9 (2012).

32. Zhang, Y. et al. Model-based analysis of ChIP-Seq (MACS). Genome Biol. 9, $\mathrm{R} 137$ (2008).

33. Phanstiel, D. H., Boyle, A. P., Araya, C. L. \& Snyder, M. P. Sushi.R: flexible, quantitative and integrative genomic visualizations for publication-quality multipanel figures. Bioinformatics 30, 2808-2810 (2014). 
34. Jeffares, D. C. et al. The genomic and phenotypic diversity of Schizosaccharomyces pombe. Nat Genet 47, 235-241 (2015).

35. McKenna, A. et al. The Genome Analysis Toolkit: a MapReduce framework for analyzing next-generation DNA sequencing data. Genome Res. 20, 1297-1303 (2010).

36. Van der Auwera, G. A. et al. From FastQ data to high confidence variant calls: the Genome Analysis Toolkit best practices pipeline. Curr Protoc Bioinformatics 43, 11.10.1-33 (2013).

37. McLaren, W. et al. The Ensembl Variant Effect Predictor. Genome Biol. 17, 122 (2016).

38. Talevich, E., Shain, A. H., Botton, T. \& Bastian, B. C. CNVkit: Genome-Wide Copy Number Detection and Visualization from Targeted DNA Sequencing.

39. Fletcher, S. J., Boden, M., Mitter, N. \& Carroll, B. J. SCRAM: a pipeline for fast 2670-2672 (2018). 


\section{Acknowledgments}

387 We thank Lorenza Di Pompeo and Andreas Fellas for laboratory support, Pin Tong 388 and Ryan Ard for sharing technical expertise and members of the Allshire lab for 389 valuable discussions. We are grateful to Adrian Bird, Wendy Bickmore and Lucia

390 Massari for comments on the manuscript. We thank Takeshi Urano for kindly

391 providing the 5.1.1 (H3K9me) antibody and Yoshinori Watanabe for the pRAD21

392 plasmid. S.T-G. was supported by the Darwin Trust of Edinburgh. R.C.A. is a

393 Wellcome Principal Research Fellow (095021, 200885); the Wellcome Centre for Cell

394 Biology is supported by core funding from Wellcome (203149).

\section{Author contributions}

396 S.T-G., P.N.C.B.A. and R.C.A. conceived the project. S.T-G. and P.N.C.B.A.

397 performed preliminary studies. S.T-G. performed experiments and bioinformatics.

398 M.S. and A.L.P. contributed to ChIP-seq and qChIP experiments. S.A.W. contributed

399 to sRNA-seq experiments. S.T-G., A.L.P. and R.C.A. wrote the manuscript.

400 Competing interests

401 The authors declare no competing interests.

402

403 
Supplementary Information Table 1. Summary of epigenetic (H3K9me2 domains) and genetic (SNPs, indels and copy number variation) changes found in unstable (UR) caffeine-resistant isolates.

\begin{tabular}{|c|c|c|c|c|}
\hline \multirow[t]{2}{*}{ Isolate } & \multicolumn{2}{|c|}{$\begin{array}{l}\text { Ectopic heterochromatin } \\
\text { location }\end{array}$} & \multirow[t]{2}{*}{ SNPs or indels in coding sequences? } & \multirow[t]{2}{*}{$\begin{array}{c}\text { Partial duplication of } \\
\text { Chr III? }\end{array}$} \\
\hline & ncRNA.394 & other loci & & \\
\hline UR-1 & & $\checkmark(h b a 1)$ & Clr5-Q264STOP / Meu27-S100Y & \\
\hline UR-2 & $\checkmark$ & & Sdo1-R11C & \\
\hline UR-3 & & $\checkmark($ ppr4) & Clr5-Q264STOP / Meu27-S100Y & \\
\hline UR-4 & & $\checkmark(g r t 1)$ & - & $\checkmark$ \\
\hline UR-5 & & $\checkmark(f i o 1)$ & Clr5-Q264STOP / Meu27-S100Y & $\checkmark$ \\
\hline UR-6 & & $\checkmark(m b x 2)$ & - & $\checkmark$ \\
\hline UR-7 & & $\checkmark$ (ppr4) & CIr5-Q264STOP / Meu27-S100Y & \\
\hline UR-8 & $\checkmark$ & & - & \\
\hline UR-9 & $\checkmark$ & & - & \\
\hline UR-10 & $\checkmark$ & & Cob1-F318L & \\
\hline UR-11 & $\checkmark$ & & - & \\
\hline UR-12 & $\checkmark$ & & - & \\
\hline UR-13 & $\checkmark$ & & - & $\checkmark$ \\
\hline UR-14 & $\checkmark$ & & Npp-W300STOP / SPBC16H5.13-S1011L & $\checkmark$ \\
\hline UR-15 & $\checkmark$ & & - & $\checkmark$ \\
\hline UR-16 & $\checkmark$ & & - & \\
\hline UR-17 & $\checkmark$ & & SPCC777.02-R120R & $\checkmark$ \\
\hline UR-18 & $\checkmark$ & & SPCC777.02-R120R & $\checkmark$ \\
\hline UR-19 & $\checkmark$ & & Sdo1-R11C & $\checkmark$ \\
\hline UR-20 & $\checkmark$ & & - & \\
\hline UR-21 & $\checkmark$ & & - & $\checkmark$ \\
\hline UR-22 & $\checkmark$ & & - & $\checkmark$ \\
\hline UR-23 & $\checkmark$ & & Pch1-Q234STOP & \\
\hline UR-24 & $\checkmark$ & & - & \\
\hline UR-25 & $\checkmark$ & & - & $\checkmark$ \\
\hline UR-26 & $\checkmark$ & & SPBC1271.08c-A133A & \\
\hline UR-27 & $\checkmark$ & & SPCC4B3.13-A229V & \\
\hline UR-28 & $\checkmark$ & & Mug72-N116S & \\
\hline UR-29 & $\checkmark$ & & Mug72-N116S & \\
\hline UR-30 & $\checkmark$ & & - & \\
\hline
\end{tabular}




\section{Supplementary Information Table 2. Schizosaccharomyces pombe strains used}

\section{in this study.}

\begin{tabular}{|c|c|c|}
\hline Strain number & Name & Description \\
\hline 143 & wt & $h$ - ED972 wild-type \\
\hline B4411 & SR-1 & Stable 16 mM Caffeine Resistant Isolate - From wt - 1 \\
\hline B4412 & SR-2 & Stable 16 mM Caffeine Resistant Isolate - From wt - 2 \\
\hline B4413 & UR-1 & Unstable 16 mM Caffeine Resistant Isolate - From wt - 1 \\
\hline B4414 & UR-2 & Unstable 16 mM Caffeine Resistant Isolate - From wt - 2 \\
\hline B4415 & UR-3 & Unstable 16 mM Caffeine Resistant Isolate - From wt - 3 \\
\hline B4416 & UR-4 & Unstable 16 mM Caffeine Resistant Isolate - From wt - 4 \\
\hline B4417 & UR-5 & Unstable 16 mM Caffeine Resistant Isolate - From wt - 5 \\
\hline B4418 & UR-6 & Unstable 16 mM Caffeine Resistant Isolate - From wt - 6 \\
\hline B4419 & UR-7 & Unstable 16 mM Caffeine Resistant Isolate - From wt - 7 \\
\hline B4420 & UR-8 & Unstable 16 mM Caffeine Resistant Isolate - From wt - 8 \\
\hline B4421 & UR-9 & Unstable 16 mM Caffeine Resistant Isolate - From wt - 9 \\
\hline B4422 & UR-10 & Unstable 16 mM Caffeine Resistant Isolate - From wt - 10 \\
\hline B4423 & UR-11 & Unstable 16 mM Caffeine Resistant Isolate - From wt - 11 \\
\hline B4424 & UR-12 & Unstable 16 mM Caffeine Resistant Isolate - From wt - 12 \\
\hline B4425 & UR-13 & Unstable $16 \mathrm{mM}$ Caffeine Resistant Isolate - From wt - 13 \\
\hline B4426 & UR-14 & Unstable 16 mM Caffeine Resistant Isolate - From wt - 14 \\
\hline B4427 & UR-15 & Unstable 16 mM Caffeine Resistant Isolate - From wt - 15 \\
\hline B4428 & UR-16 & Unstable 16 mM Caffeine Resistant Isolate - From wt - 16 \\
\hline B4429 & UR-17 & Unstable 16 mM Caffeine Resistant Isolate - From wt - 17 \\
\hline B4430 & UR-18 & Unstable 16 mM Caffeine Resistant Isolate - From wt - 18 \\
\hline B4431 & UR-19 & Unstable 16 mM Caffeine Resistant Isolate - From wt - 19 \\
\hline B4432 & UR-20 & Unstable 16 mM Caffeine Resistant Isolate - From wt - 20 \\
\hline B4433 & UR-21 & Unstable 16 mM Caffeine Resistant Isolate - From wt - 21 \\
\hline B4434 & UR-22 & Unstable 16 mM Caffeine Resistant Isolate - From wt - 22 \\
\hline B4435 & UR-23 & Unstable 16 mM Caffeine Resistant Isolate - From wt - 23 \\
\hline B4436 & UR-24 & Unstable 16 mM Caffeine Resistant Isolate - From wt - 24 \\
\hline B4437 & UR-25 & Unstable 16 mM Caffeine Resistant Isolate - From wt - 25 \\
\hline B4438 & UR-26 & Unstable 16 mM Caffeine Resistant Isolate - From wt - 26 \\
\hline B4439 & UR-27 & Unstable 16 mM Caffeine Resistant Isolate - From wt - 27 \\
\hline B4440 & UR-28 & Unstable $16 \mathrm{mM}$ Caffeine Resistant Isolate - From wt - 28 \\
\hline B4441 & UR-29 & Unstable 16 mM Caffeine Resistant Isolate - From wt - 29 \\
\hline B4442 & UR-30 & Unstable 16 mM Caffeine Resistant Isolate - From wt - 30 \\
\hline B4443 & SR-1 c/r4 $\Delta-1$ & SR-1 clr4D::NAT - transformant 1 \\
\hline B4444 & SR-1 c/r4 $\Delta-2$ & SR-1 clr4 $\triangle:: N A T$ - transformant 2 \\
\hline B4445 & SR-1 NAT control - 1 & SR-1 NAT:3' of ura4 - transformant 1 \\
\hline B4446 & SR-1 NAT control - 2 & SR-1 NAT:3' of ura4 - transformant 2 \\
\hline B4447 & SR-2 c/r4 $\Delta-1$ & SR-2 clr4 $\triangle:: N A T$ - transformant 1 \\
\hline B4448 & SR-2 clr4 $\Delta-2$ & SR-2 c/r4 $\triangle:: N A T$ - transformant 2 \\
\hline B4449 & SR-2 NAT control - 1 & SR-2 NAT:3' of ura4 - transformant 1 \\
\hline B4450 & SR-2 NAT control - 2 & SR-2 NAT:3' of ura4 - transformant 2 \\
\hline B4451 & UR-1 c/r4 $\Delta-1$ & UR-1 clr4 $:: N A T$ - transformant 1 \\
\hline B4452 & UR-1 c/r4 $\Delta-2$ & UR-1 clr4 $\triangle:: N A T$ - transformant 2 \\
\hline B4453 & UR-1 NAT control-1 & UR-1 NAT:3' of ura4 - transformant 1 \\
\hline B4454 & UR-1 NAT control-2 & UR-1 NAT:3' of ura4 - transformant 2 \\
\hline B4455 & UR-2 c/r4 $\Delta$ - 1 & UR-2 clr4A::NAT - transformant 1 \\
\hline B4456 & UR-2 c/r4 $\Delta-2$ & UR-2 clr4A::NAT - transformant 2 \\
\hline B4457 & UR-2 NAT control - 1 & UR-2 NAT:3' of ura4 - transformant 1 \\
\hline B4458 & UR-2 NAT control - 2 & UR-2 NAT:3' of ura4 - transformant 2 \\
\hline B4352 & Pap1-N424STOP & $h$ - pap1-N424STOP \\
\hline B4459 & UR-2 + 14 days -CAF & UR-2 after growth on -CAF media for 14 days \\
\hline B4460 & hba1s & $h-h b a 1 \triangle:: N A T$ \\
\hline B4461 & SPBC17G9.12c $\triangle$ & $h-S P B C 17 G 9.12 c \triangle \because N A T$ \\
\hline B4462 & $n c R N A .393 \Delta$ & $h-n c R N A .393 \Delta:: N A T$ \\
\hline
\end{tabular}




\begin{tabular}{|c|c|c|}
\hline B4463 & ncRNA.394A & $h-n c R N A .394 \Delta:: N A T$ \\
\hline B4464 & eno1014 & $h$ - eno101 $\triangle:: N A T$ \\
\hline B3797 & TetR-C/r4* & $h+l e u 1+a d h 21-T e t R O F F-2 x F L A G-C / r 4-c d d$ \\
\hline B3808 & $4 x t e t O-I I$ & h-4xtetO 3' of SPBC17G9.13c leu1-32 \\
\hline B3813 & $4 x t e t O-I$ & h- 4xtetO 5' of hba1 leu1-32 \\
\hline B3820 & $4 x t e t O-I I I$ & $h-4 x t e t O 5$ of ura4 leu1-32 \\
\hline B4465 & TetR-Clr4* $+4 x t e t O-I I$ & $\begin{array}{l}\text { h+ leu1+adh21-TetROFF-2xFLAG-Clr4-cdd 4xtetO 3' of } \\
\text { SPBC17G9.13c }\end{array}$ \\
\hline B4466 & TetR-Clr4* $+4 x t e t O-I$ & $h+$ leu1+adh21-TetROFF-2xFLAG-Clr4-cdd 4xtetO 5' of hba1 \\
\hline B4467 & TetR-Clr4* + 4xtetO-IIII & $h+$ leu1+adh21-TetROFF-2xFLAG-Clr4-cdd x4tetO 5' of ura4 \\
\hline B4468 & UR-2 (7 days $+\mathrm{CAF})$ & UR-2 after growth on +CAF media for 3 days \\
\hline B4469 & $\begin{array}{l}\text { UR-2 ( } 7 \text { days }+ \text { CAF } \\
\rightarrow 14 \text { days }- \text { CAF) }\end{array}$ & $\begin{array}{l}\text { UR- } 2 \text { after growth on +CAF media for } 3 \text { days and then on -CAF } \\
\text { media for } 14 \text { days }\end{array}$ \\
\hline
\end{tabular}




\section{Supplementary Information Table 3. Oligonucleotides used in this study.}

\begin{tabular}{|c|c|c|}
\hline Name & Sequence & Description \\
\hline qAct1-F & GGTTTCGCTGGAGATGATG & $\mathrm{qPCR} \mathrm{act}^{+}-\mathrm{F}$ \\
\hline qAct1-R & ATACCACGCTTGCTTTGAG & qPCR act $^{+}-\mathrm{R}$ \\
\hline qDg-F & AATTGTGGTGGTGTGGTAATAC & qPCR $d g$ repeats $-\mathrm{F}$ \\
\hline qDg-R & GGGTTCATCGTTTCCATTCAG & qPCR $d g$ repeats - $R$ \\
\hline ST-52 & GAATTGTGGAGCCATGTCCC & qPCR slu $7^{+}-\mathrm{F}$ \\
\hline ST-53 & TCTTCTCCTGTCCAACGAGC & qPCR slu7 $7^{+}-\mathrm{R}$ \\
\hline ST-872 & GAAACCCAGAAATTCGCAGGT & qPCR kin17 $7^{+}-\mathrm{F}$ - primer pair 1 hba1 locus \\
\hline ST-873 & ATGAGTTGCTTGGGCATCCA & qPCR kin $17^{+}-\mathrm{R}$ - primer pair 1 hba1 locus \\
\hline ST-62 & CAGCAAATTGGGGACTGTGT & qPCR ish $1^{+}-\mathrm{F}$ - primer pair 2 hba1 locus \\
\hline ST-63 & CTCAAGAAGCCTGGGAGTCA & qPCR ish $1^{+}-\mathrm{R}$ - primer pair 2 hba1 locus \\
\hline ST-64 & CGATGATCTGGTTGTATGGTGG & qPCR $h b a 1^{+}-\mathrm{F}$ - primer pair 3 hba1 locus \\
\hline ST-65 & TGCTCAGTACGCCATCTTGA & qPCR $h b a 1^{+}-\mathrm{R}$ - primer pair 3 hba1 locus \\
\hline ST-66 & GGGCTATCCTTAACGCTCTTC & qPCR $h b a 1^{+} c d s-F-$ primer pair 4 hba1 locus \\
\hline ST-67 & CGCCTCCTCTGAACCAAAAG & qPCR $h b a 1^{+} c d s-R-$ primer pair 4 hba1 locus \\
\hline ST-58 & CTTCCCACATCGCGTTCATT & qPCR alp4 $4^{+}-\mathrm{F}$ - primer pair 5 hba1 locus \\
\hline ST-59 & ACCTAAATCATCGCTGCTGG & qPCR alp4 $4^{+}-\mathrm{R}$ - primer pair 5 hba1 locus \\
\hline ST-393 & GGGCATGACAATCTCCGACT & qPCR pyr1+ - F - primer pair 1 ncRNA.394 locus \\
\hline ST-394 & GGCCTACCTCGGTGATCTTG & qPCR pyr1+ - R - primer pair 1 ncRNA.394 locus \\
\hline ST-401 & CCGTATGGTGAAGCAGGGTT & qPCR SPBC17G9.12c ${ }^{+}-\mathrm{F}$ - primer pair 2 ncRNA.394 locus \\
\hline ST-402 & CCCGATCTCCGTGTAAGCAA & qPCR SPBC17G9.12c $c^{+}-\mathrm{R}$ - primer pair 2 ncRNA.394 locus \\
\hline ST-184 & TTCGTCGTATGCCCTCTTGC & qPCR SPBC17G9.13c $c^{+}-\mathrm{F}$ - primer pair 3 ncRNA.394 locus \\
\hline ST-185 & AAAATCCGCCATTTGCCCAG & qPCR SPBC17G9.13c ${ }^{+}$- R - primer pair 3 ncRNA.394 locus \\
\hline ST-251 & TGCTGTAGTGATGCAGAGGAG & qPCR $n c R N A .393^{+}-\mathrm{F}$ - primer pair 4 ncRNA.394 locus \\
\hline ST-252 & GCGGCCATTTTGTTTACATTCC & qPCR $n c R N A .393^{+}$- R - primer pair 4 ncRNA.394 locus \\
\hline ST-190 & GAAAATTAGCGCGGCCGTTA & qPCR $n c R N A .394^{+}$- F - primer pair 5 ncRNA.394 locus \\
\hline ST-191 & TCAATCTGCTTGTCCCACCC & qPCR $n c R N A .394^{+}$- R - primer pair 5 ncRNA.394 locus \\
\hline ST-263 & GTGCTGCCCAAAAGAAGCTC & qPCR eno101 ${ }^{+}$- F - primer pair 6 ncRNA.394 locus \\
\hline ST-264 & TGGGAACCACCGTTCAAGAC & qPCR eno101+ - R - primer pair 6 ncRNA.394 locus \\
\hline ST-249 & AGCTTTCAAGGTAGCGGGTG & $\mathrm{qPCR}_{\text {cut }}{ }^{+}-\mathrm{F}$ \\
\hline ST-250 & TTCCTCTGCTCAGCGTAGAC & qPCR cut2 ${ }^{+}-\mathrm{R}$ \\
\hline PA-354 & CAGTTAGTTTCAGGTTTCCC & $\mathrm{qPCR}+2.5 \mathrm{~kb}$ ura $^{+}$- $\mathrm{F}$ - primer pair 1 ura 4 locus \\
\hline PA-355 & GCAGAGTAATGGTGATTGG & $\mathrm{qPCR}+2.5 \mathrm{~kb}$ ura4 ${ }^{+}-\mathrm{R}$ - primer pair 1 ura4 locus \\
\hline ST-874 & CACACAGTTTCAGAAGAAC & qPCR tam14+ - F - primer pair 2 ura4 locus \\
\hline ST-875 & GTTACGAGGAATCTTGGTAG & qPCR $\operatorname{tam} 14^{+}-\mathrm{R}$ - primer pair 2 ura4 locus \\
\hline ST-796 & CGCGACTGACAAGTTGCTTT & qPCR ura4 $^{+}-\mathrm{F}$ - primer pair 3 ura4 locus \\
\hline ST-797 & AGCTAGAGCTGAGGGGATGA & qPCR ura4 $^{+}-\mathrm{R}$ - primer pair 3 ura4 locus \\
\hline ST-800 & TGGTTTAAATCAAATCTTCCATGCG & qPCR 5' of ura $^{+}-\mathrm{F}$ - primer pair 4 ura4 locus \\
\hline ST-801 & TGAGCAAACTGCTTTTGTGGT & qPCR 5' of ura $^{+}$- R - primer pair 4 ura4 locus \\
\hline ST-788 & GGATGAAGCTGTCTCCCTGG & qPCR new $25^{+}-\mathrm{F}$ - primer pair 5 ura4 locus \\
\hline ST-789 & TATTGCTGCTTCTTCCCTGGC & qPCR new $25^{+}-\mathrm{R}$ - primer pair 5 ura4 locus \\
\hline ST-876 & GGAATCTATGTCGTTGCCG & qPCR pmp20 $0^{+}$F - primer pair 6 ura4 locus \\
\hline ST-877 & GTAAACTCTCCGTTCCAGTC & qPCR pmp $20^{+}-\mathrm{R}$ - primer pair 6 ura4 locus \\
\hline Clr4-KO-F & $\begin{array}{l}\text { ATTTTTTAAATTCGTTCAGGCA } \\
\text { TCATTTGGAGGGTTTGCTAAA } \\
\text { AATCATCTCACCAAACAAGAG } \\
\text { GTTATTAGTITTGGACGGAT } \\
\text { CCCCGGGTTAATTAA }\end{array}$ & $\mathrm{KO}$ of $\mathrm{clr} 4^{+}$with Bahler construct - F \\
\hline Clr4-KO-R & $\begin{array}{l}\text { AAATGAATGACCTTTTTCAGTT } \\
\text { TAACAGTAATGGAGAAAAACA } \\
\text { AATTGTAATTATTGGAGTCAAC } \\
\text { CAGTAATAAATTAGCGAATTC } \\
\text { GAGCTCGTTTAAAC }\end{array}$ & $\mathrm{KO}$ of $\mathrm{c} / \mathrm{r} 4^{+}$with Bahler construct - R \\
\hline ST-3 & $\begin{array}{l}\text { GTCCAACACCCAGTTGTTAAC } \\
\text { TGCTTATAATGACGCGTATGAT } \\
\text { TGCGATATTITAAGACTCTGGC } \\
\text { CATCCACCGCTTTATCCGACG } \\
\text { GATCCCCGGGTTAATTAA }\end{array}$ & Inserting natMX6 marker 3' of $\mathrm{ura4}^{+}$(Control) - F \\
\hline ST-12 & $\begin{array}{l}\text { GCAGGTTCTAGTAATGCGCAT } \\
\text { TCAATTTGTAGTATTCTTAAATA }\end{array}$ & Inserting natMX6 marker 3' of $\mathrm{ura}^{+}$(Control) - R \\
\hline
\end{tabular}


ATCATTAAACGACAAGGGCCTT CCGTGCTATAGTGTGAATTCGA GCTCGTTTAAAC

\begin{tabular}{|c|c|c|}
\hline ST-866 & $\begin{array}{l}\text { CtagaGGTCTCgGACTCTCCATTTTCGT } \\
\text { TAGAATTAGTTTCGAGACCcttCC }\end{array}$ & Golden Gate cloning pap1-sgRNA-1-F \\
\hline ST-867 & $\begin{array}{l}\text { GGaagGGTCTCgAAACTAATTCTAACG } \\
\text { AAAATGGAGAGTCcGAGACCtctaG }\end{array}$ & Golden Gate cloning pap1-sgRNA-1-R \\
\hline ST-868 & $\begin{array}{l}\text { AGCATGGCGCGAACCCGCTGAATCA } \\
\text { TTGGACAAAGAATTCTTTAACGACGA } \\
\text { GGGTGAAATAGATGATGTTTTTCATAA } \\
\text { TTATTTTCATAATTCTAACGTC }\end{array}$ & Pap1-N424STOP - HR template - F \\
\hline ST-869 & $\begin{array}{l}\text { GCTCAGGGAATGATTCGTTGGCATTC } \\
\text { TCCAGAAAATCAAGACCATGCAATGA } \\
\text { ATTAGTGATCAAGTCTCCATITTCGTT } \\
\text { AGACGTTAGAATTATGAAAAT }\end{array}$ & Pap1-N424STOP - HR template - R \\
\hline ST-284 & $\begin{array}{l}\text { CAGCTGTGTGTTTGATTGAATCCACA } \\
\text { TTCGTCCTCATGTACTCATAGCTAGG } \\
\text { TGAAATATATTAGGCTTCAGTGATTC } \\
\text { GCGGATCCCCGGGTTAATTAA }\end{array}$ & $\mathrm{KO}$ of $h b a 1^{+}$with Bahler construct - F \\
\hline ST-285 & $\begin{array}{l}\text { GAATGAATAAGAACCATAGTGAAGA } \\
\text { GCTAAAAAAGAATCGAAAAGTACTT } \\
\text { ACTATTTACGAGTGGATCTTCTATC } \\
\text { TCGCGAATTCGAGCTCGTTTAAAC }\end{array}$ & $\mathrm{KO}$ of $h b a 1^{+}$with Bahler construct - R \\
\hline ST-391 & $\begin{array}{l}\text { TCTTCTGCCTAACCATACTACTTCTT } \\
\text { CTAGCCTTCAGACTTAAAAGCTTCG } \\
\text { CCTTTAGAAAACATCTCTATTCCTTC } \\
\text { AAACGGATCCCCGGGTTAATTAA }\end{array}$ & $\mathrm{KO}$ of $S P B C 17 G 9.12 c^{+}$with Bahler construct - $\mathrm{F}$ \\
\hline ST-392 & $\begin{array}{l}\text { CAAGAGAGATGGAAAACAGAGGA } \\
\text { ATTGTGAACGTTCTCCTTATTCATAT } \\
\text { TTCCATAAAGCTTCTCCAATGACCTT } \\
\text { TATTGGAATTCGAGCTCGTTTAAAC }\end{array}$ & $\mathrm{KO}$ of $S P B C 17 G 9.12 C^{+}$with Bahler construct - F \\
\hline ST-307 & $\begin{array}{l}\text { GATAAAATCTTAGAGATTGTTGCTA } \\
\text { AATAAGCAAACAGTGTCTTTGCTGT } \\
\text { AACTGGTGAGATATGTTTAAAATTAAA } \\
\text { TCACGGATCCCCGGGTTAATTAA }\end{array}$ & $\mathrm{KO}$ of $n c R N A .393^{+}$with Bahler construct - F \\
\hline ST-308 & $\begin{array}{l}\text { TGATATAATATATTTTCCTTCTTTACT } \\
\text { ATTACATTTCCTATTTTTTCACCATTT } \\
\text { ACGATATGTGTAACACTATCTAACCC } \\
\text { GAATTCGAGCTCGTTTAAAC }\end{array}$ & $\mathrm{KO}$ of $n c R N A .393^{+}$with Bahler construct - R \\
\hline ST-95 & $\begin{array}{l}\text { TAATGAAAAAGGTTGCTAATTGGTTT } \\
\text { GTTATATAAGAGTATGTCGCATTTGT } \\
\text { TTACGATAGGAGAGAGCGATTTTCC } \\
\text { ACACGGATCCCCGGGTTAATTAA }\end{array}$ & KO of $n c R N A .394^{+}$with Bahler construct - F \\
\hline ST-96 & $\begin{array}{l}\text { TATTACTATGACTCTGGTTCTAGCTC } \\
\text { GACTCTGACCCTTGCCTGACATACA } \\
\text { AATACTTTGCTCTITTCAAAATGTACC } \\
\text { GTGAATTCGAGCTCGTTAAAC }\end{array}$ & $\mathrm{KO}$ of $n c R N A .394^{+}$with Bahler construct - $\mathrm{R}$ \\
\hline ST-305 & $\begin{array}{l}\text { ATATATAGAGTGGAAGGGCCGTCCG } \\
\text { TTAGGACTTGTTTCAGTAAGAATCAAT } \\
\text { TAGTATTCTACAGTAAACATCGTTAAT } \\
\text { CCGGATCCCCGGGTTAATTAA }\end{array}$ & $\mathrm{KO}$ of eno101+ with Bahler construct - F \\
\hline ST-306 & $\begin{array}{l}\text { CTACTTCTACTACAACAACAGTTTAC } \\
\text { TTTAATACTAATAATAAATAAACACG } \\
\text { CAACCTGGCAAATTAATCCAAAACG } \\
\text { CAAGAATTCGAGCTCGTTTAAAC }\end{array}$ & $\mathrm{KO}$ of eno $101^{+}$with Bahler construct - R \\
\hline ST-756 & $\begin{array}{l}\text { CtagaGGTCTCgGACTGGTGCTTGACT } \\
\text { TCTAATCTTGTTTCGAGACCcttCC }\end{array}$ & Golden Gate cloning 4xtetO-I-sgRNA-F \\
\hline ST-757 & $\begin{array}{l}\text { GGaagGGTCTCgAAACAAGATTAGAAG } \\
\text { TCAAGCACCAGTCcGAGACCtctaG }\end{array}$ & Golden Gate cloning 4xtetO-I-sgRNA-R \\
\hline ST-732 & AAACGCTAATCTAGCATGTCATGAAGG & Making 4tetO-I-HR-template - 1F \\
\hline ST-733 & $\begin{array}{l}\text { actagtaggccttgCCGTATTGAAATCAAAA } \\
\text { TTATTAATAATGAGTAAGTGAATATATA } \\
\text { CCA }\end{array}$ & Making 4tetO-I-HR-template - 1R \\
\hline ST-734 & $\begin{array}{l}\text { TGATTTCAATACGGcaaggcctactagtgcat } \\
\text { gca }\end{array}$ & Making 4tetO-I-HR-template - $2 \mathrm{~F}$ \\
\hline ST-735 & $\begin{array}{l}\text { TCTATAACTTTTACGTTAGctggatttcgttt } \\
\text { acctcaccac }\end{array}$ & Making 4tetO-I-HR-template - 2R \\
\hline ST-736 & $\begin{array}{l}\text { gaggtaaacgaaatccagCTAACGTAAAAGT } \\
\text { TATAGACAGTATTATAACAAGTATTATT } \\
\text { GTAAAA }\end{array}$ & Making 4tetO-I-HR-template - 3F \\
\hline
\end{tabular}




\begin{tabular}{|c|c|c|}
\hline ST-737 & $\begin{array}{l}\text { TTTAATTGTATTTTTTTATTCAAAGGTTC } \\
\text { TACTTTGTCAATCATTTTCAA }\end{array}$ & Making 4tetO-I-HR-template - 3R \\
\hline ST-752 & $\begin{array}{l}\text { CtagaGGTCTCgGACTATTTCTTTTG } \\
\text { CTTACGGTCGTTTCGAGACCcttCC }\end{array}$ & Golden Gate cloning 4xtetO-II-sgRNA-F \\
\hline ST-753 & $\begin{array}{l}\text { GGaagGGTCTCgAAACGACCGTAA } \\
\text { AGCAAAAGAAATAGTCcGAGACCtctaG }\end{array}$ & Golden Gate cloning 4xtetO-II-sgRNA-R \\
\hline ST-720 & $\begin{array}{l}\text { TTGAATTAATTCATAGAGTATGATAAAA } \\
\text { ATTGATAGTAAATTCATTGG }\end{array}$ & Making 4tetO-II-HR-template - 1F \\
\hline ST-721 & $\begin{array}{l}\text { cactagtaggccttgATGCATGCTAATAAA } \\
\text { TCATCGTAACTCAAGTAG }\end{array}$ & Making 4tetO-II-HR-template - 1R \\
\hline ST-722 & $\begin{array}{l}\text { TTTATTAGCATGCATcaaggcctactagtgc } \\
\text { atgca }\end{array}$ & Making 4tetO-II-HR-template - 2F \\
\hline ST-723 & $\begin{array}{l}\text { TTTTTTTTTTCATAAATATTTActgga } \\
\text { tttcgtttacctcaccacc }\end{array}$ & Making 4tetO-II-HR-template - 2R \\
\hline ST-724 & $\begin{array}{l}\text { tggtgaggtaaacgaaatccagTAAATATTTAT } \\
\text { GAAAAAAAAAATAAATGATTCATAACAA } \\
\text { GCAGATGAAAA }\end{array}$ & Making 4tetO-II-HR-template - 3F \\
\hline ST-725 & $\begin{array}{l}\text { TTTGTAATGTATAATCTTCATTTATTTT } \\
\text { GAAGAGTCCTAATTCGT }\end{array}$ & Making 4tetO-II-HR-template - 3R \\
\hline ST-760 & $\begin{array}{l}\text { CtagaGGTCTCgGACTATATTTTAGATA } \\
\text { GTTCTGTGGTTCGAGACCcttCC }\end{array}$ & Golden Gate cloning 4xtetO-III-sgRNA-F \\
\hline ST-761 & $\begin{array}{l}\text { GGaagGGTCTCgAAACCACAGAACTAT } \\
\text { CTAAAATATAGTCcGAGACCtctaG }\end{array}$ & Golden Gate cloning 4xtetO-III-sgRNA-R \\
\hline ST-744 & CGGTAAGAAAACACGACATGTGCAG & Making 4tetO-III-HR-template - 1F \\
\hline ST-745 & $\begin{array}{l}\text { catgcactagtaggccttgTATAATTAAGATG } \\
\text { TTTAGAGACTTATACAATTTTGTCTTT } \\
\text { ATAAATTCT }\end{array}$ & Making 4tetO-III-HR-template - 1R \\
\hline ST-746 & $\begin{array}{l}\text { CTAAAACATCTTAATTATAcaaggccta } \\
\text { ctagtgcatgca }\end{array}$ & Making 4tetO-III-HR-template - 2F \\
\hline ST-747 & $\begin{array}{l}\text { TTTGCACTTTGTGAATctggatttcgttt } \\
\text { acctcaccacca }\end{array}$ & Making 4tetO-III-HR-template - 2R \\
\hline ST-748 & $\begin{array}{l}\text { gtaaacgaaatccagATTCACAAAGTGC } \\
\text { AAACATTATCATGAAAAAGAAC }\end{array}$ & Making 4tetO-III-HR-template - 3F \\
\hline ST-749 & $\begin{array}{l}\text { TGAAAAAGATAATCAGCCTTATAATC } \\
\text { TTTACAAAAGTAAGAAATTCT }\end{array}$ & Making 4tetO-III-HR-template - 3R \\
\hline
\end{tabular}



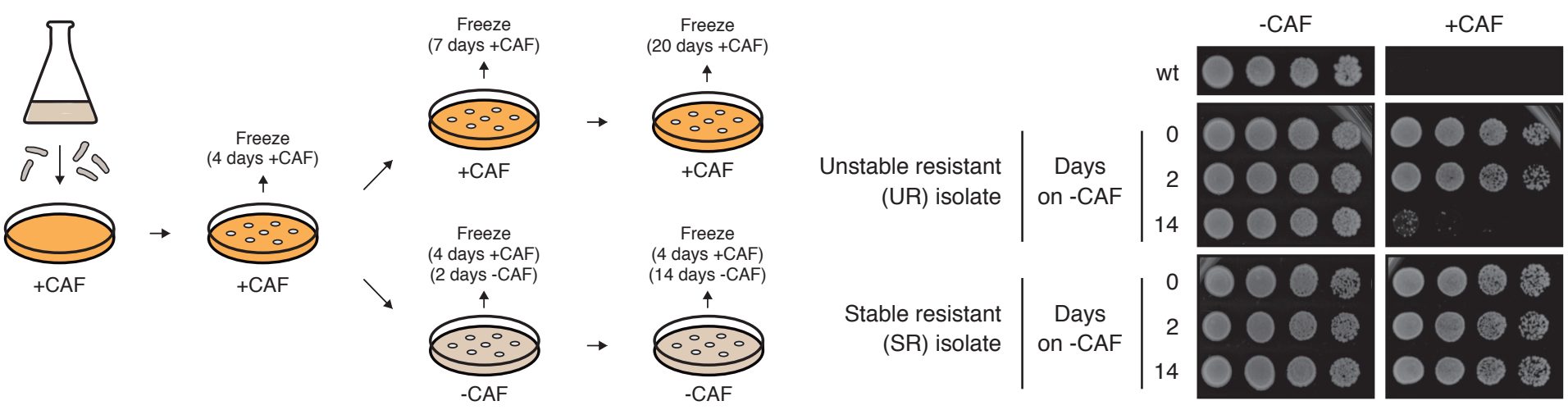

C

d

Total analyzed resistant isolates $(n=176)$
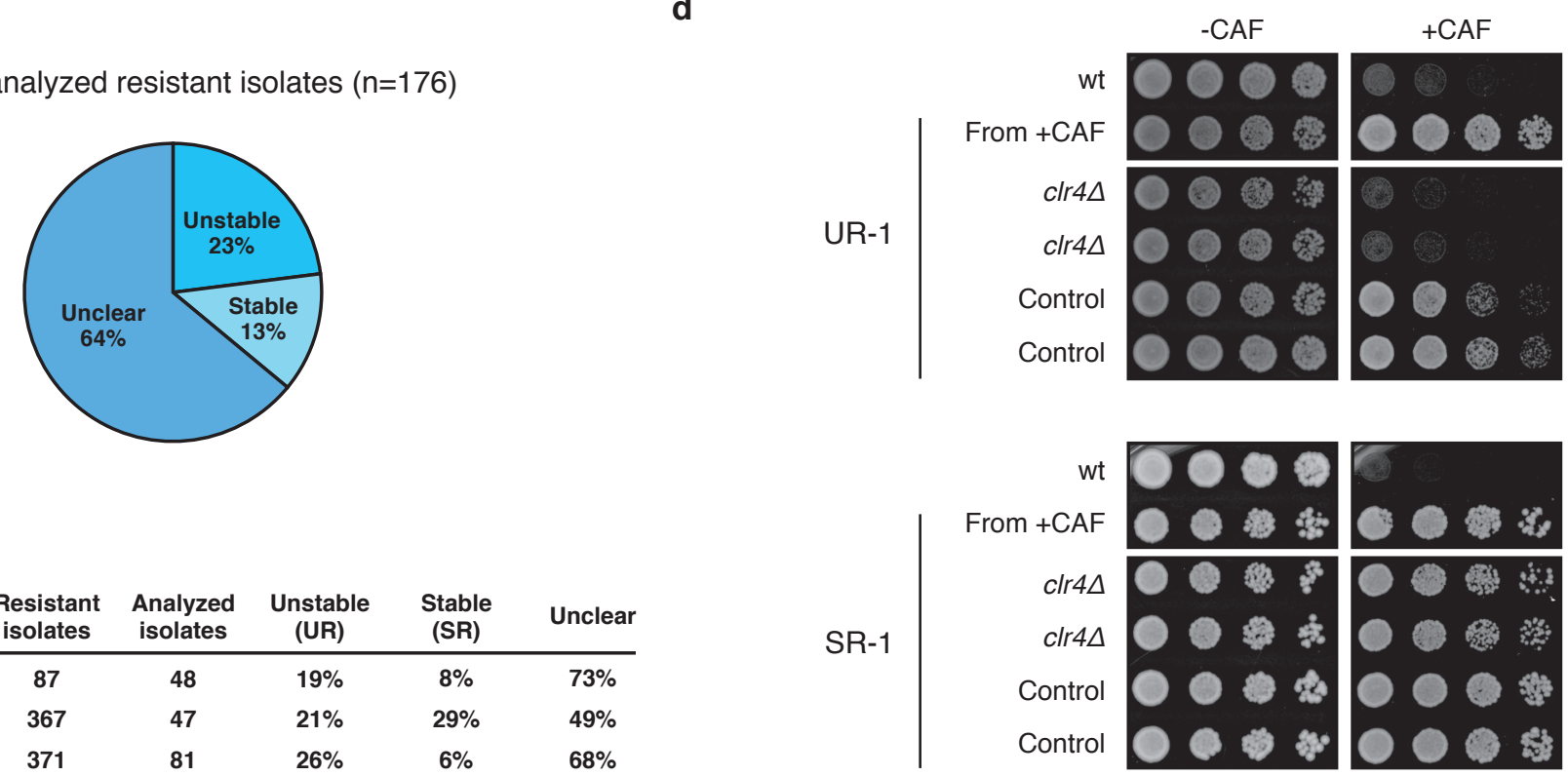

Figure 1. Identification of heterochromatin-dependent epimutants resistant to caffeine

a, Schematic of the screening strategy. S. pombe wild-type (wt) cells were plated on caffeine-containing (+CAF) plates. Caffeine-resistant isolates were then grown on $+\mathrm{CAF}$ plates for 4,7 or 20 days or on non-selective (-CAF) medium plates for 2 and 14 days. Cells were then serially diluted and spotted on -CAF and +CAF media to assess resistance to caffeine.

b, Unstable (UR) and stable (SR) caffeine-resistant isolates were identified. After growth on non-selective media for 14 days caffeine resistance is lost in UR isolates but not in SR isolates.

c, Frequency of unstable (UR) / stable (SR) caffeine-resistant isolates obtained from 3 independent screens. $64 \%$ of isolates did not display a clear phenotype (unclear).

d, Caffeine resistance in UR isolates depends on the Clr4 H3K9 methyltransferase. clr4+ $(\mathrm{clr} 4 \Delta)$ or an unlinked intergenic region (Control) were deleted in unstable (UR-1) and stable (SR-1) caffeine-resistant isolates. 
a ChIP-seq: H3K9me2

\section{Chr I}

Chr II

Chr III

tel1L cen1

tel1R tel2L cen2 tel2R tel3L cen3 $\stackrel{t e l 3 R}{\longrightarrow}$
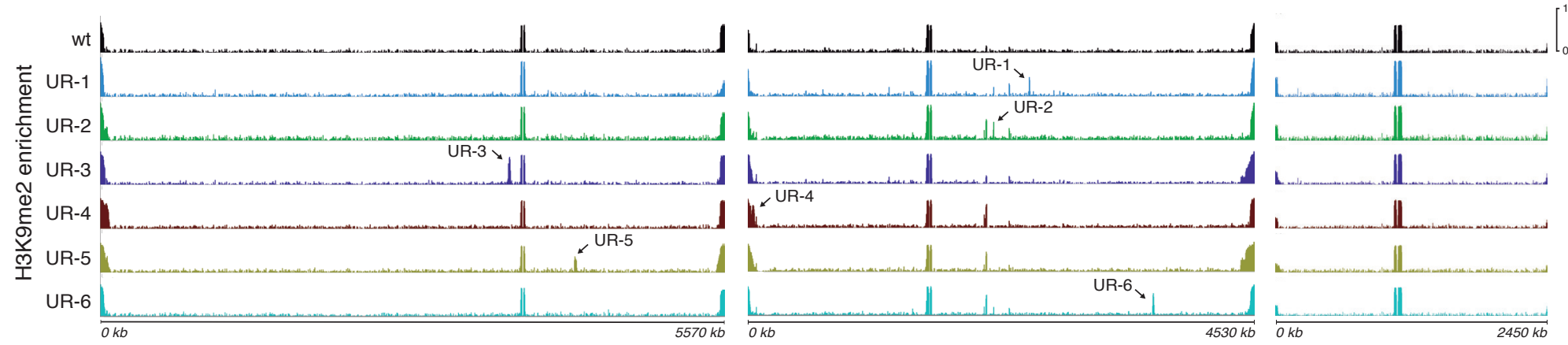

b

ChIP-seq: H3K9me2
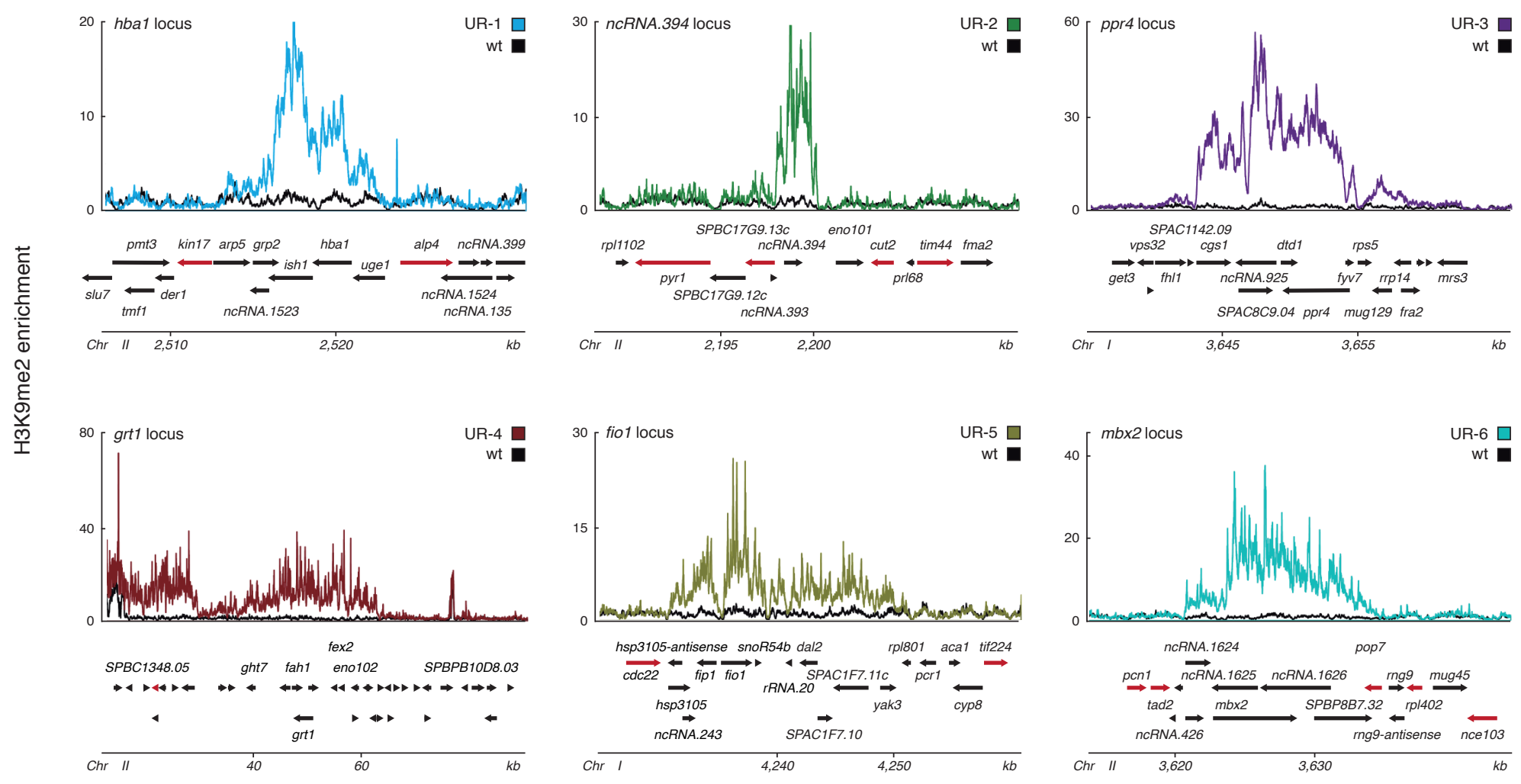

c
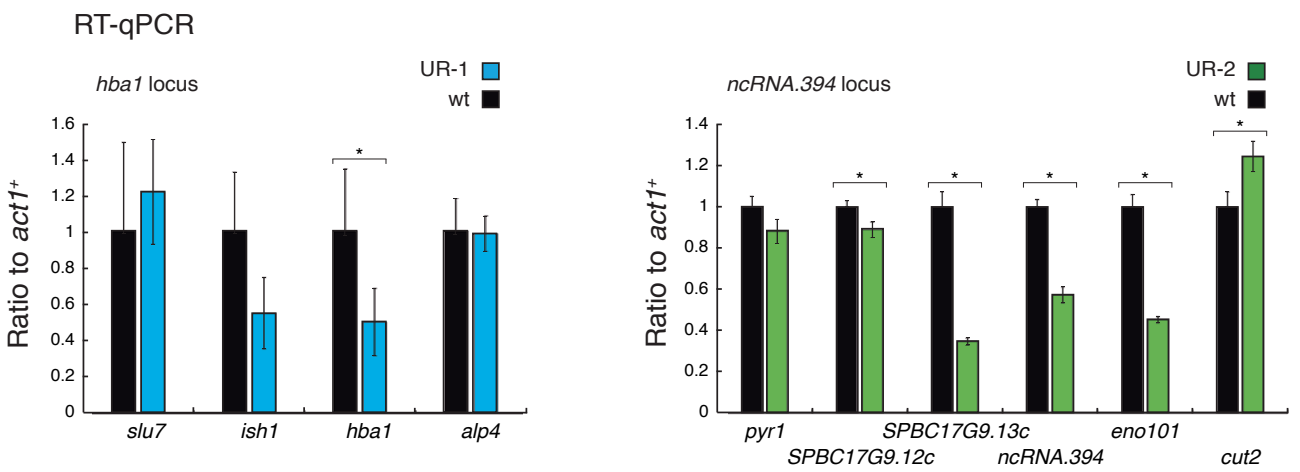

Figure 2. Ectopic domains of heterochromatin are detected in unstable (UR) caffeine-resistant isolates

a, Genome-wide H3K9me2 ChIP-seq enrichment in UR isolates and wt. Data are represented as relative fold enrichment over input.

b, H3K9me2 ChIP-seq enrichment at ectopic heterochromatin domains in individual isolates. Data are represented as relative fold enrichment over input and compared to levels in wt cells. Relevant genes within and flanking ectopic heterochromatin domains are indicated. Red arrows indicate essential genes.

c, Gene transcript levels within and flanking ectopic heterochromatin domains in isolates UR-1 and UR-2. Data are mean \pm SD (error bars) ( $\mathrm{n}=3$ experimental replicates). ${ }^{*} P<0.05$ ( $t$ test). 
a
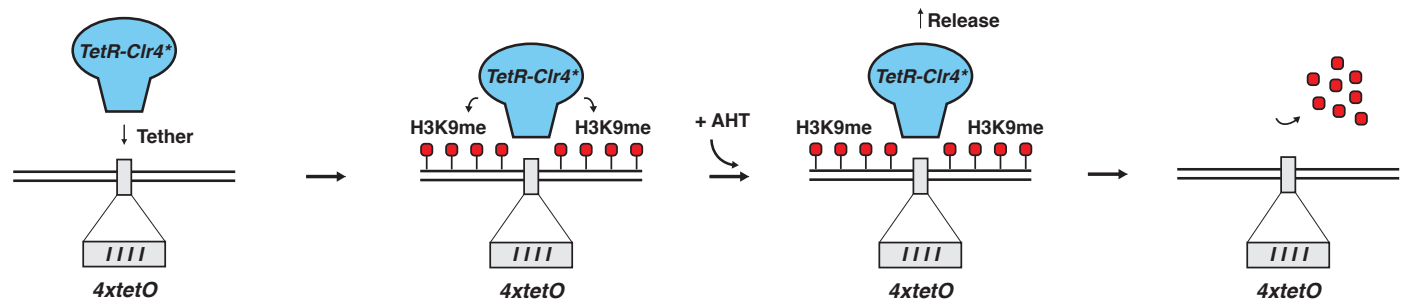

b
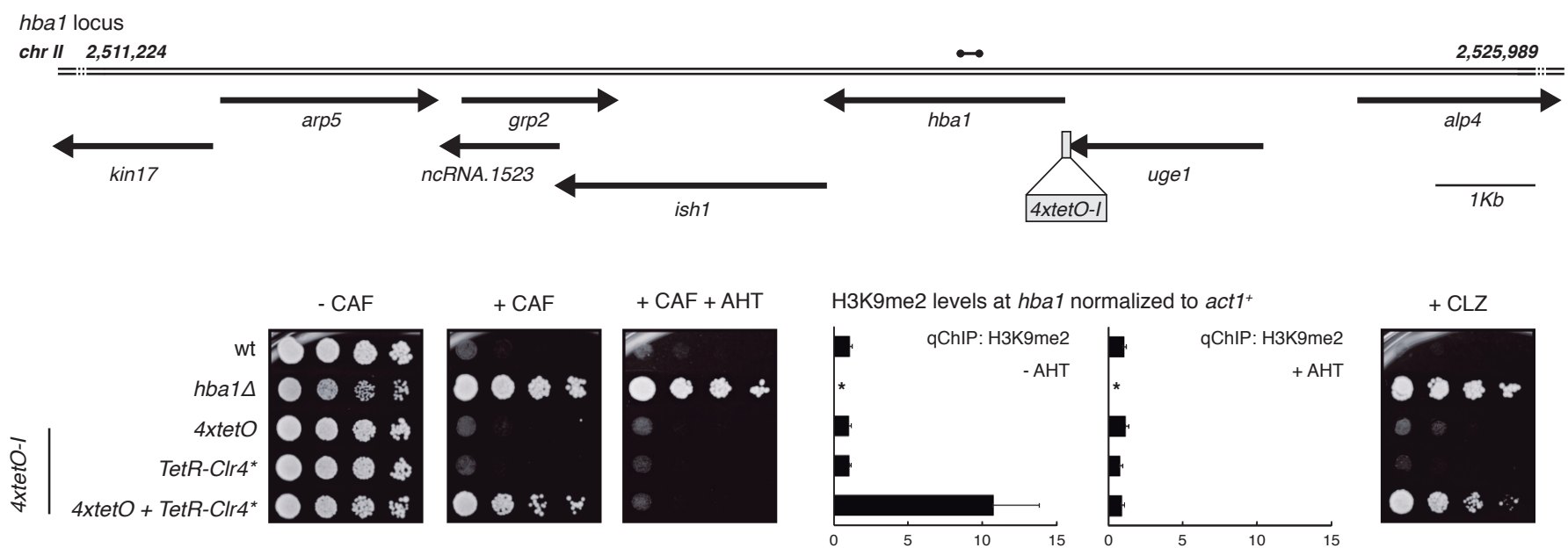

C

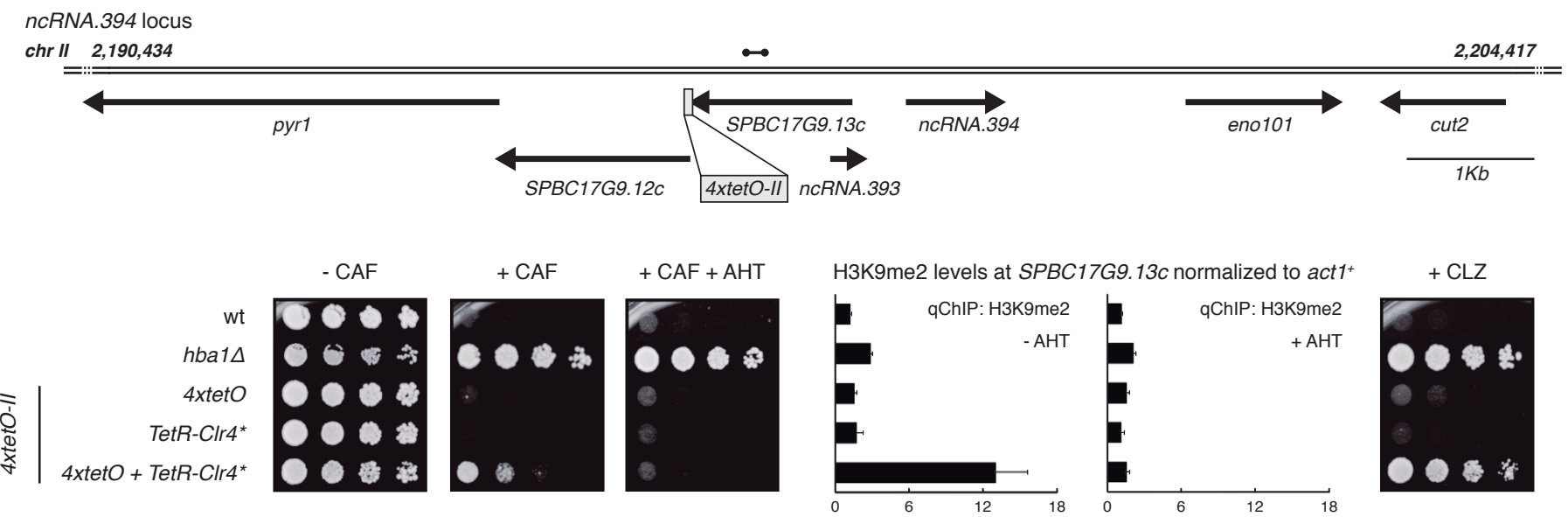

d
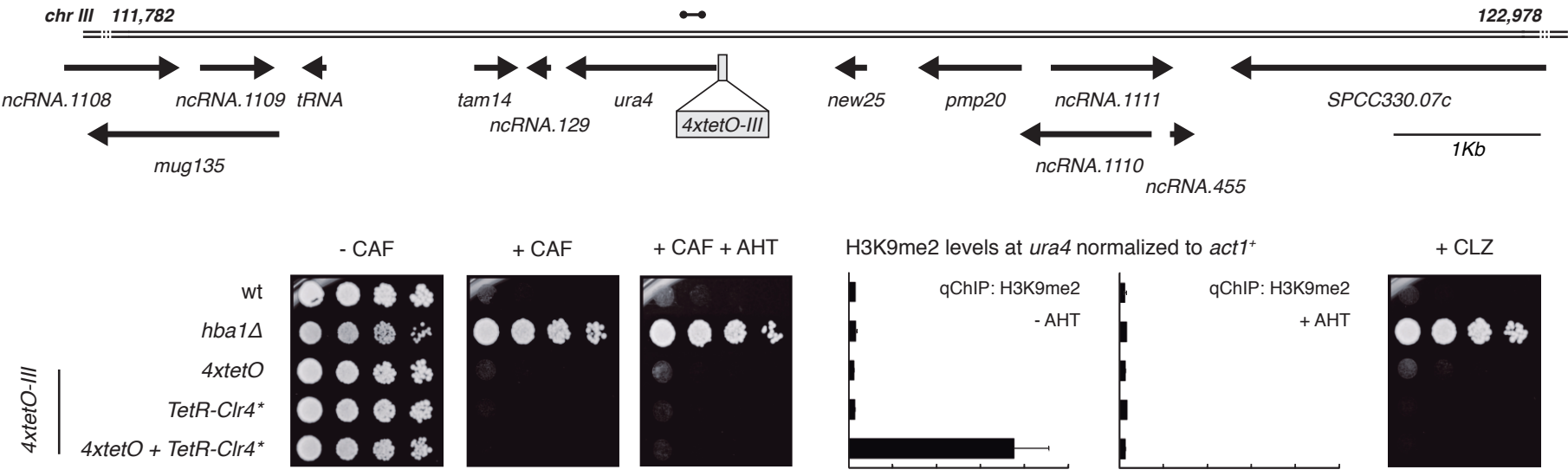

H3K9me2 levels at ura4 normalized to act $1^{+}$

$+\mathrm{CLZ}$
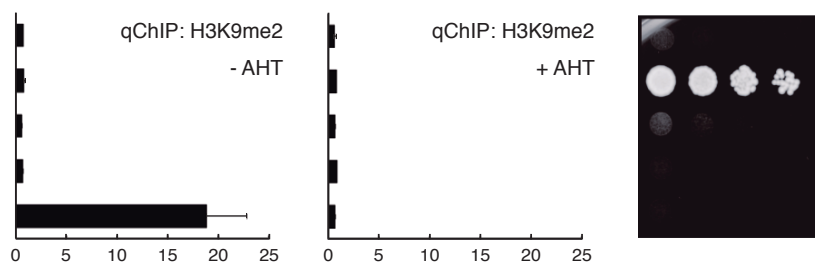

Figure 3. Forced synthetic heterochromatin placement at the identified loci is sufficient to drive caffeine resistance in wild-type cells

a, Diagram illustrating TetR-Clr4*-mediated H3K9me deposition at $4 x t e t O$ binding sites. Addition of anhydrotetracycline (+AHT) causes release of TetR-Clr4* from $4 x t e t O$ sites which results in active removal of H3K9me.

b-d, Wild-type cells harbouring $4 x$ tetO binding sites at the hba1 or ncRNA.394 loci (or ura4 as control) and expressing TetR-Clr4* were assessed for caffeine resistance in the absence or presence of AHT. Quantitative chromatin immunoprecipitation (qChIP) of H3K9me2 levels on hba1 (b), SPBC17G9.13c (c) and ura4 (d) loci. Data are mean \pm SD (error bars) $(\mathrm{n}=3$ experimental replicates). Dumbbells indicate oligonucleotides used. *Note $h b a 1$ is not present in $h b a 1 \Delta$. Strains were also assessed for resistance to the fungicide clotrimazole. 
a ChIP-Seq: H3K9me2

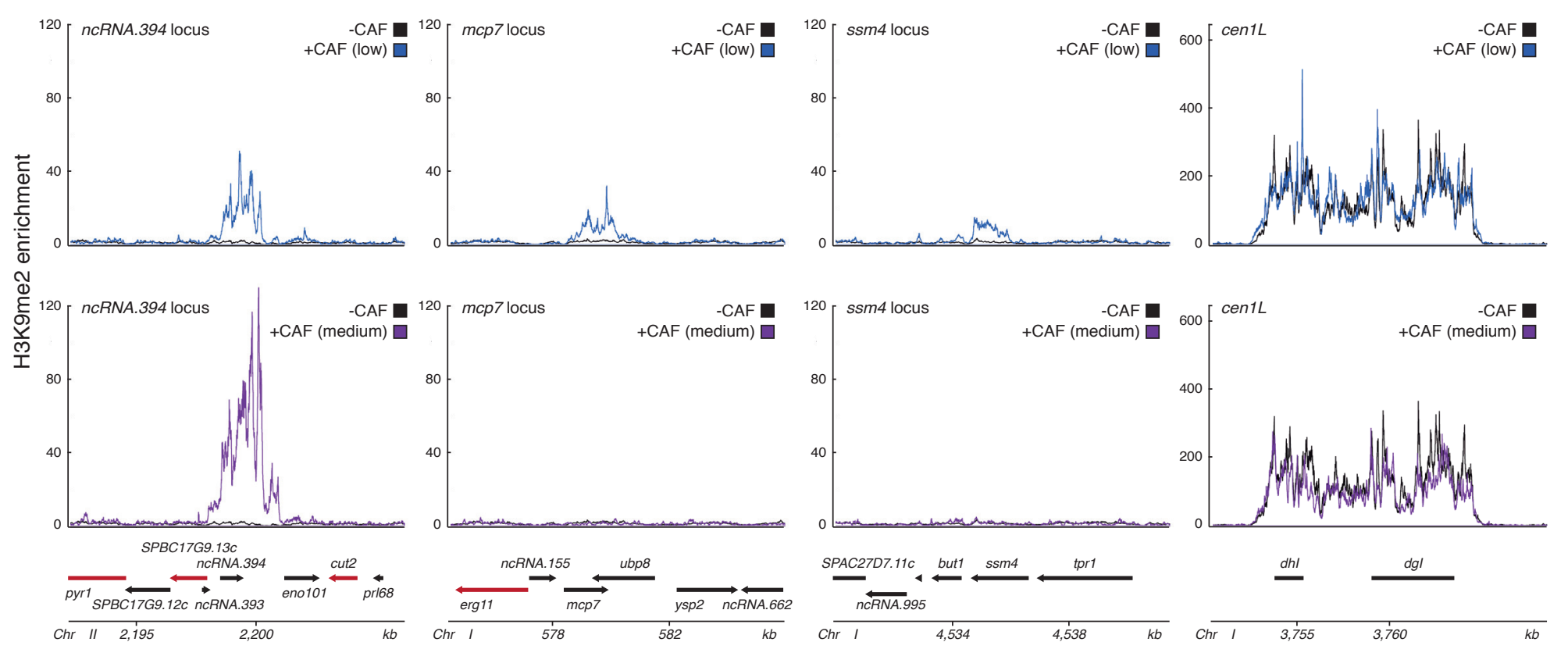

b ChIP-Seq: H3K9me2
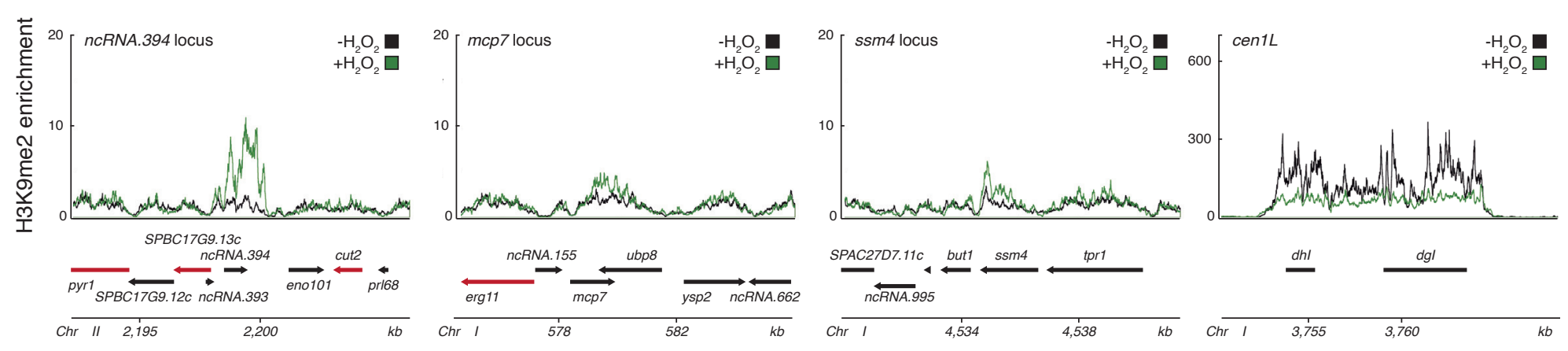

Figure 4. Dynamic heterochromatin redistribution following short exposure to external insults in wild-type cells

a, H3K9me2 ChIP-seq enrichment at ncRNA.394, mcp7 and ssm4 loci following $18 \mathrm{hr}$ exposure to low (7 mM, top) or medium (14 mM, bottom) concentrations of caffeine.

b, H3K9me2 ChIP-seq enrichment at ncRNA.394, mcp7 and ssm4 loci following 18hr exposure to a low concentration of hydrogen peroxide $(1 \mathrm{mM})$.

$\mathbf{a}-\mathbf{b}$, Data are represented as relative fold enrichment over input and compared to levels in wt cells. Relevant genes within and flanking ectopic heterochromatin domains are indicated. Red arrows indicate essential genes. H3K9me2 enrichment at pericentromeric dhl and $d g /$ repeats (cen $1 L$ ) of chromosome I shown as control (note different scale). 
a

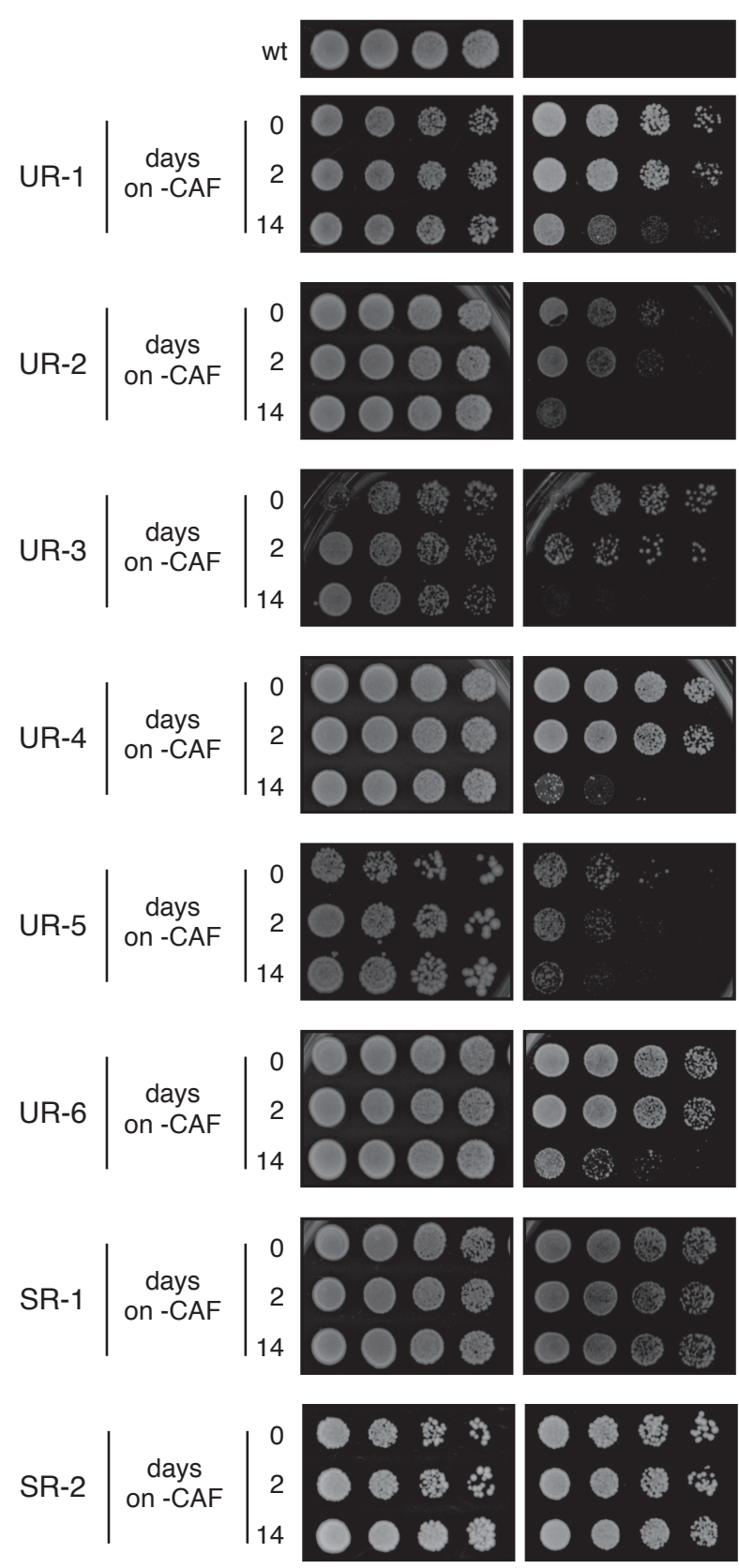

C

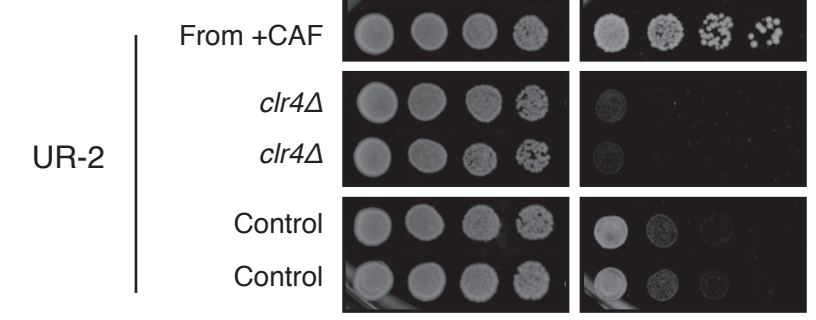

b
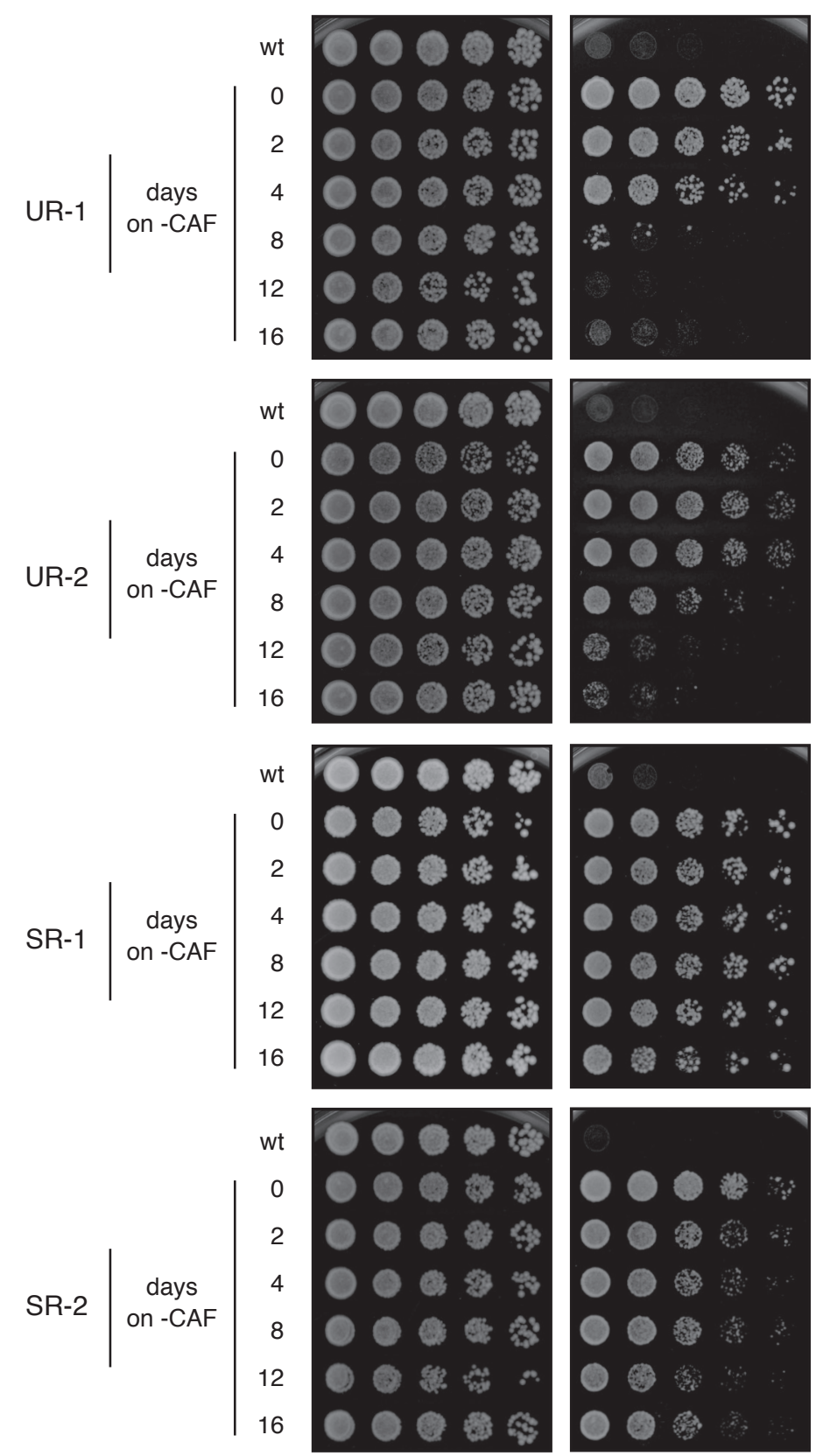

\section{Extended Data Figure 1. Identification of heterochromatin-dependent epimutants resistant to caffeine}

a, Unstable (UR) and stable (SR) caffeine-resistant isolates were identified using our screening strategy. After growth on non-selective media for 14 days caffeine resistance is lost in UR isolates but not in SR isolates.

b, Caffeine resistance is lost progressively in unstable (UR) isolates but maintained in stable (SR) isolates. 
Pap1

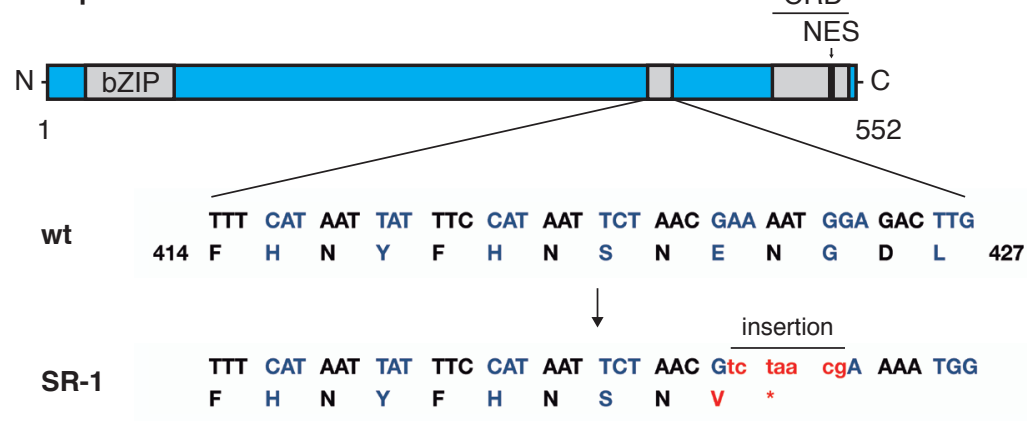

b

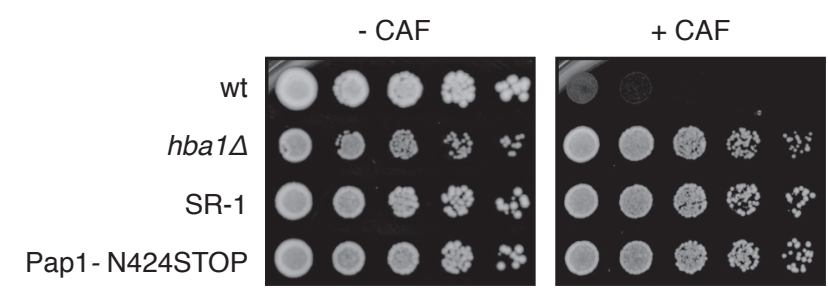

Extended Data Figure 2. A mutation in pap1+ confers caffeine resistance in the stable isolate SR-1

a, High-throughput sequencing of the stable isolate SR-1 revealed a 7-nucleotide insertion in pap1+. The insertion results in a truncated version of Pap1 (Pap1-N424STOP) lacking the Nuclear Export Signal (NES).

b, Pap1-N424STOP is resistant to caffeine. The 7-nucleotide insertion identified in SR-1 was introduced in wt cells (Pap1-N424STOP) and caffeine resistance was assessed. $h b a 1 \Delta$ and SR-1 cells were used as positive controls. 


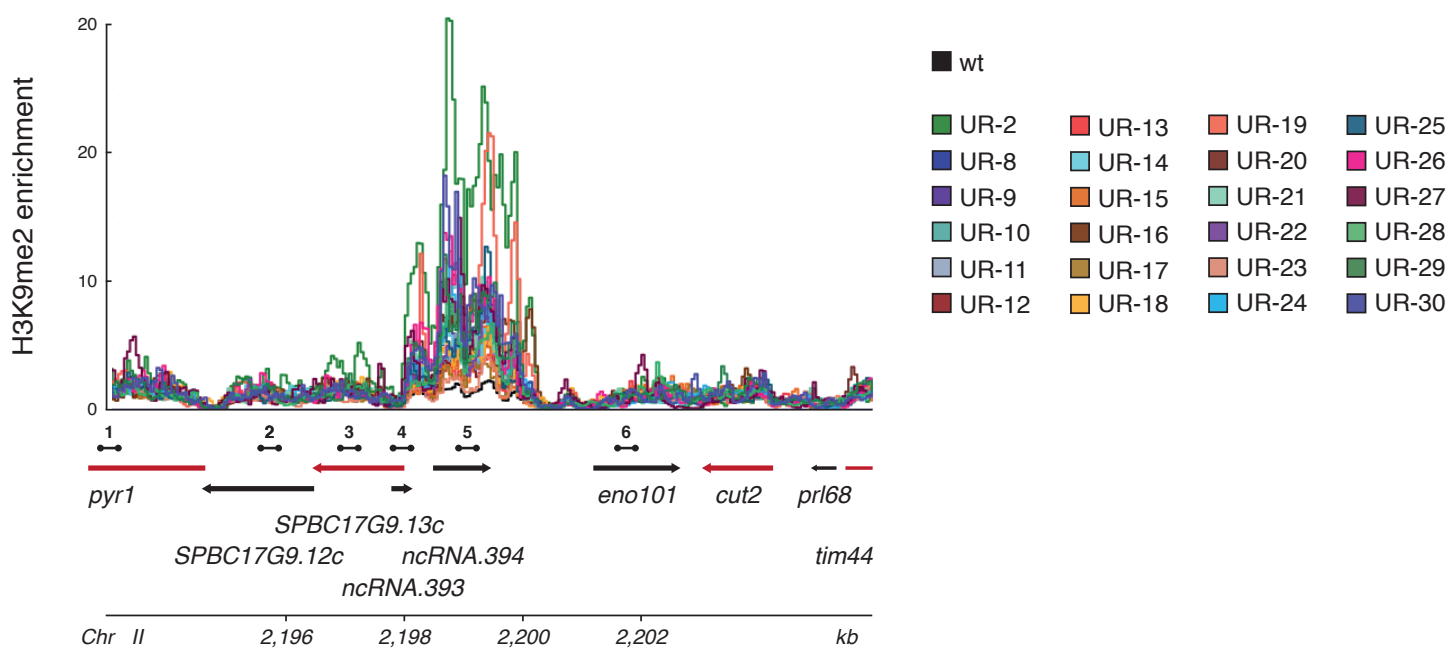

b

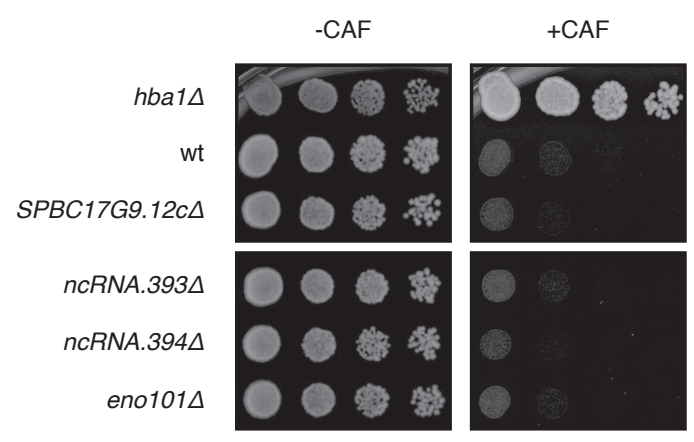

C

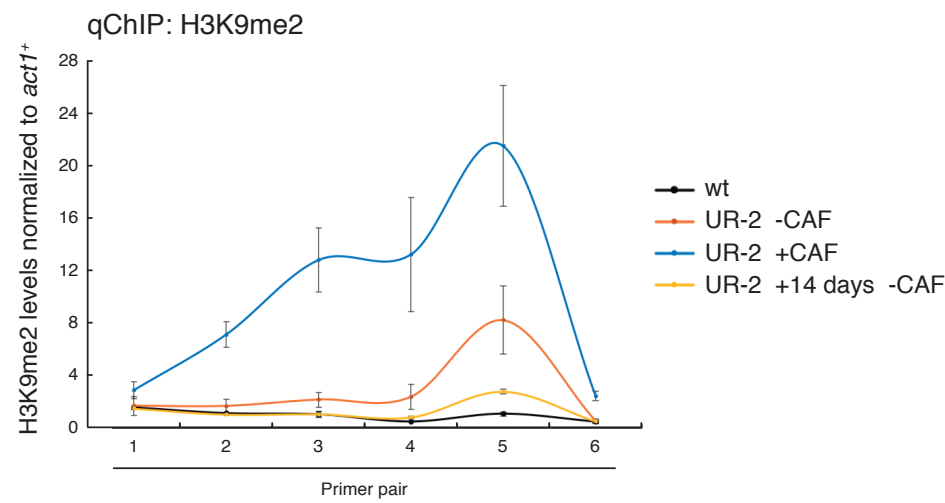

Extended Data Figure 3.24 of 30 unstable (UR) caffeine-resistant isolates present an ectopic heterochromatin domain over the ncRNA.394 locus

a, H3K9me2 ChIP-seq enrichment at the ncRNA.394 locus in individual isolates. Data are represented as relative fold enrichment over input and compared to levels in wt cells. Relevant genes within and flanking ectopic heterochromatin domains are indicated. Red arrows indicate essential genes. Dumbbells indicate oligonucleotides used in c.

b, Deletion of ncRNA.394 or non-essential adjacent genes does not result in caffeine resistance.

c, Quantitative chromatin immunoprecipitation (qChIP) of H3K9me2 levels at the ncRNA.394 locus in UR-2 cells. UR-2 cells were grown in the absence (-CAF) or presence (+CAF) of caffeine overnight or in the absence of caffeine for 14 days $(+14$ days $-\mathrm{CAF})$. Data are mean \pm $\mathrm{SD}$ (error bars) ( $\mathrm{n}=3$ experimental replicates). Oligonucleotides used are indicated in $\mathbf{a}$. 


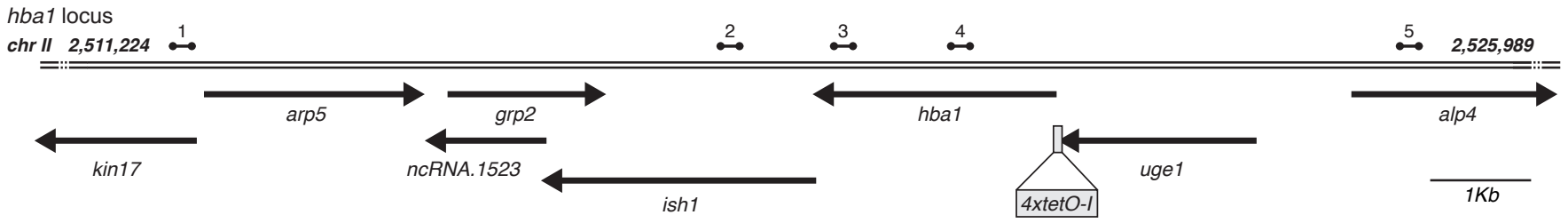

qChIP: H3K9me2
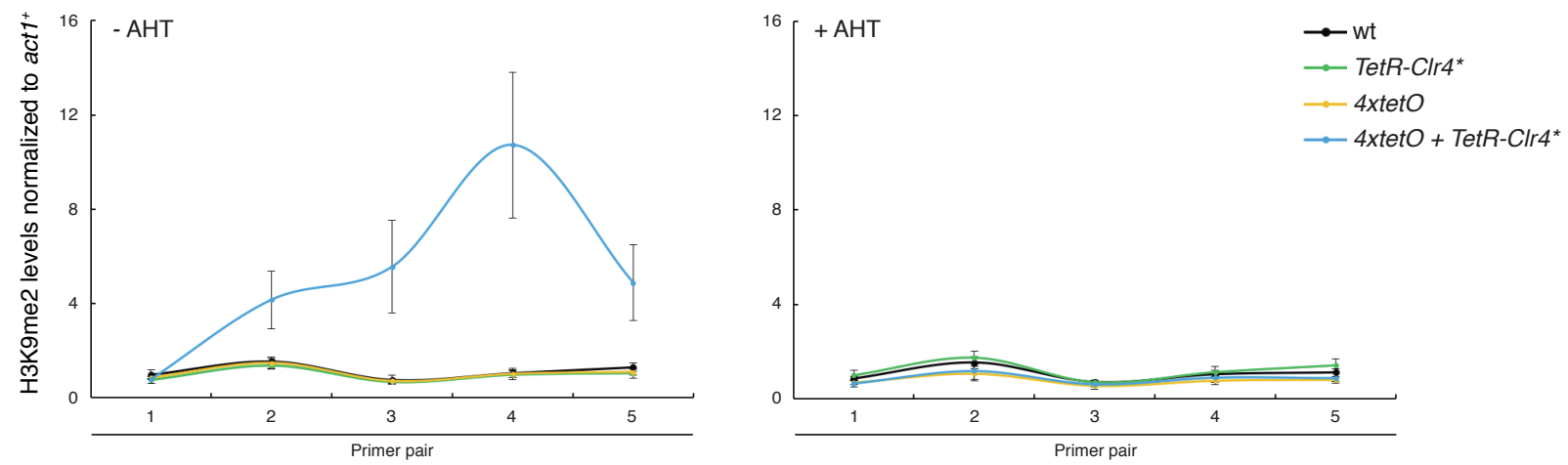

b

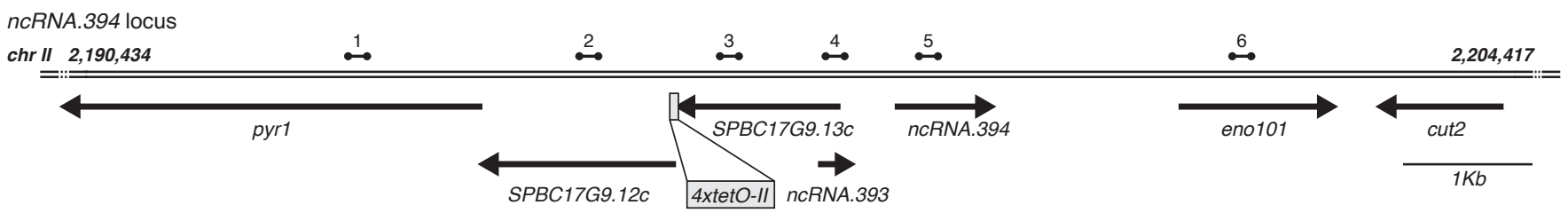

qChIP: H3K9me2
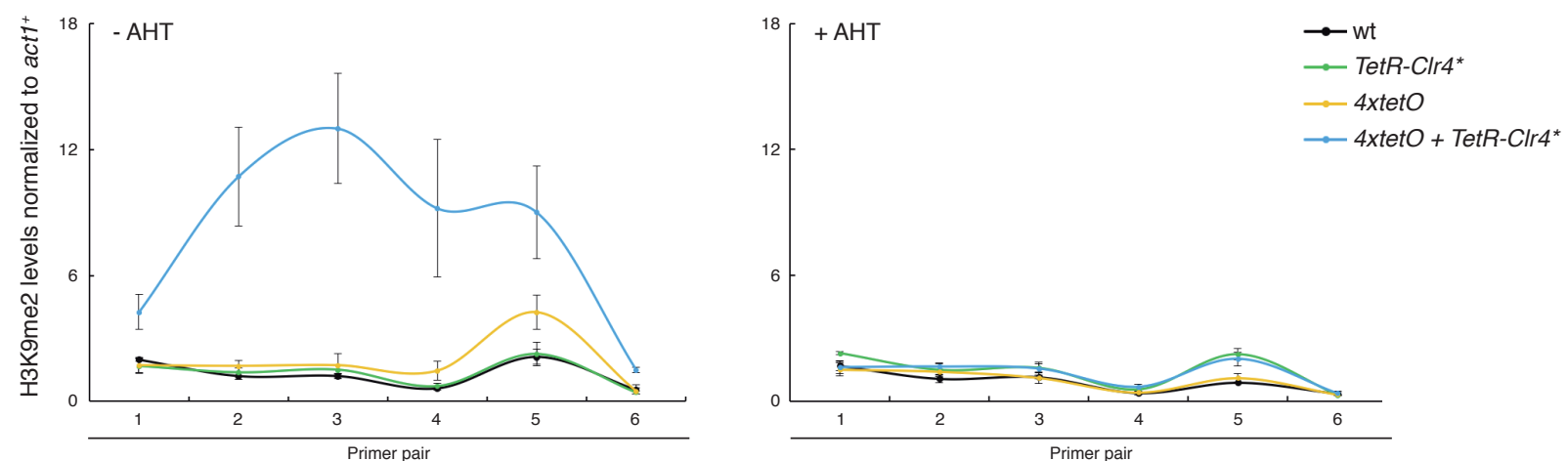

C

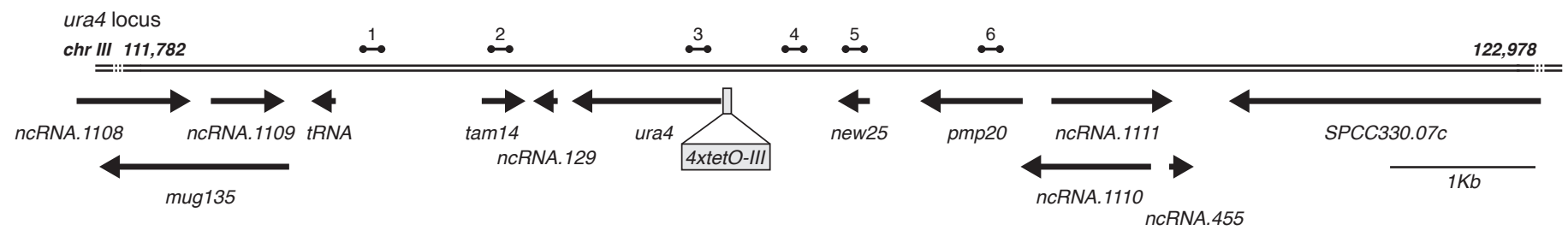

qChIP: H3K9me2
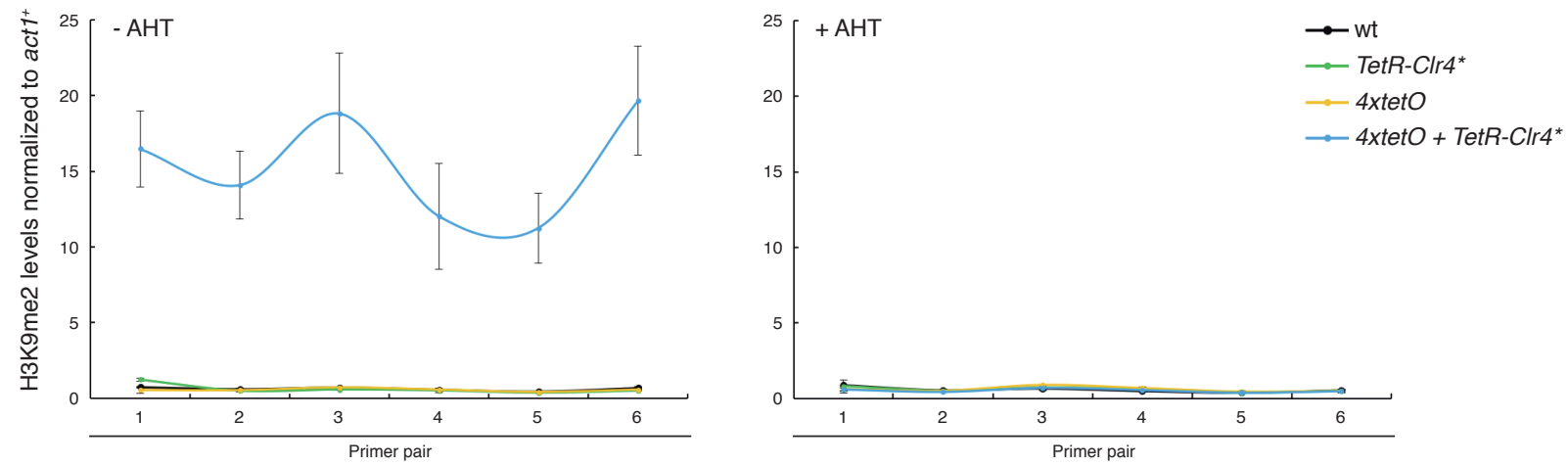

Extended Data Figure 4. Forced synthetic heterochromatin placement at the identified loci is sufficient to drive caffeine resistance in wild-type cells

a-c, Quantitative chromatin immunoprecipitation (qChIP) of $\mathrm{H} 3 \mathrm{~K} 9 \mathrm{me} 2$ levels in wild-type cells harbouring $4 x t e t O$ binding sites at the identified ectopic heterochromatin loci (or ura 4 as control) and expressing TetR-Clr4* in the absence or presence of AHT. a, hba1 locus. $\mathbf{b}$, $n c R N A .394$ locus. c, ura4 locus. Data are mean \pm SD (error bars) $(n=3$ experimental replicates). Dumbbells indicate oligonucleotides used. 
a

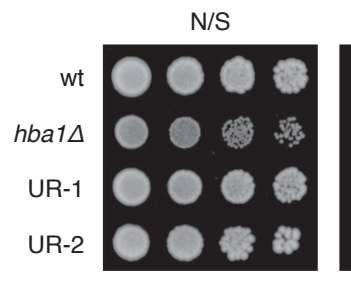

$+\mathrm{CLZ}$

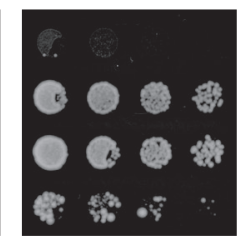

b

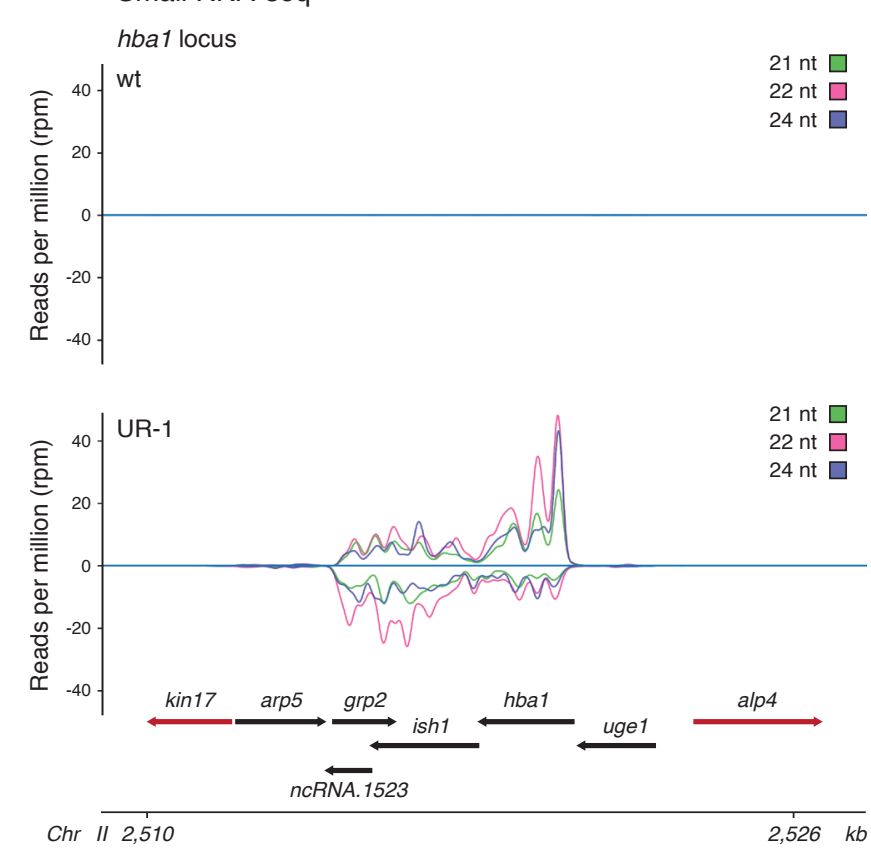

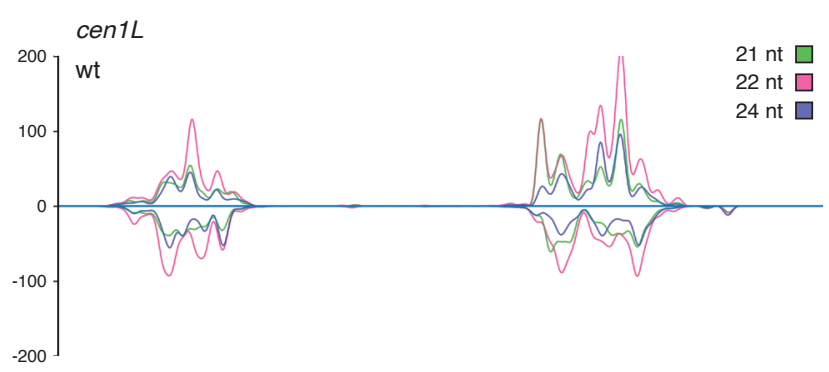

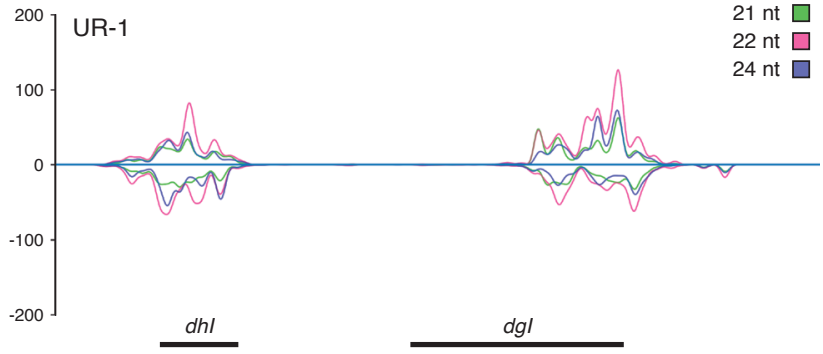

Chr $\longdiv { 3 , 7 5 3 }$

$3,766 \quad k b$

C
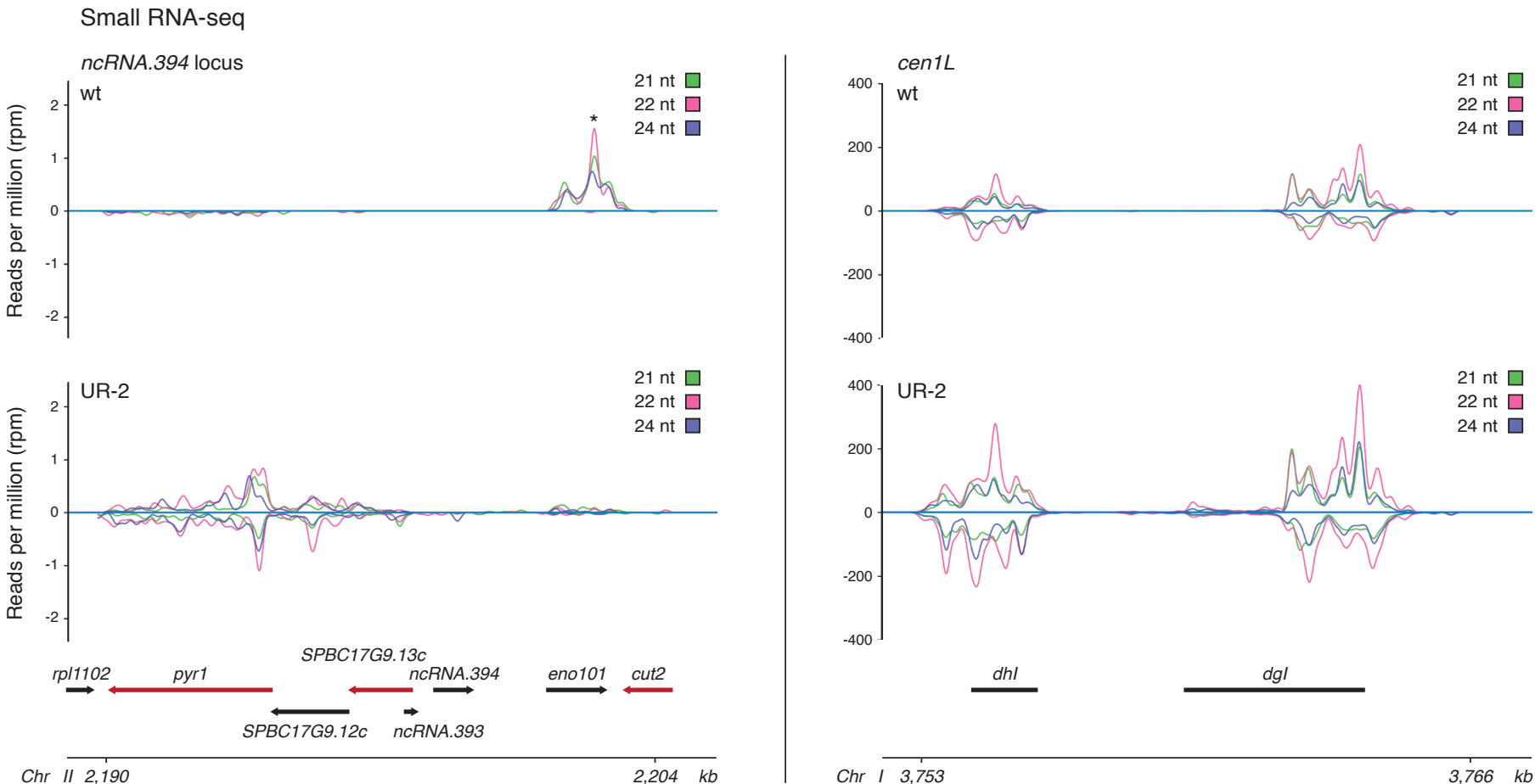

$d g l$

Chr $\longdiv { 1 } 3,753$

$3,766 \quad k b$

Extended Data Figure 5. Unstable (UR) caffeine-resistant isolates show cross-resistance to the fungicide clotrimazole and siRNA generation at ectopic heterochromatin domains

a, Unstable caffeine-resistant isolates UR-1 and UR-2 were serially diluted and spotted on non-selective (N/S), +CAF and + CLZ plates to assess resistance to caffeine and clotrimazole.

b-c, Left, small RNA sequencing showing presence of siRNAs (21-24 nucleotides) at ectopic heterochromatin domains in UR-1 (b, hba1 locus) and UR-2 (c, ncRNA.394 locus) cells compared to wt cells. Right, pericentromeric siRNAs mapping to $d h /$ and $d g /$ repeats (cen1L) of chromosome I shown as control. Experiments were performed twice with similar results. *Transcripts mapping to the highly expressed gene eno101+ in euchromatic wt conditions (note these are unidirectional RNAs and not siRNAs). 
a
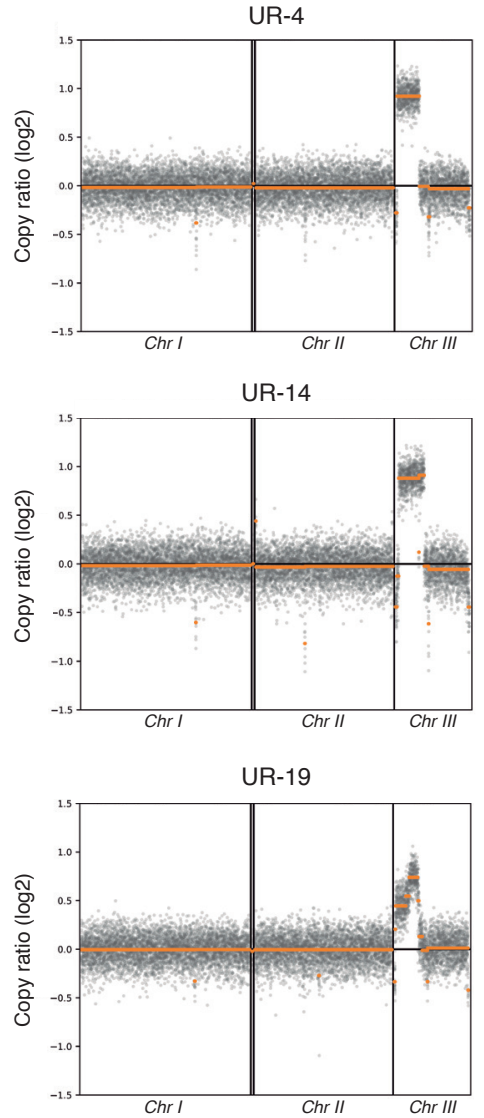

b

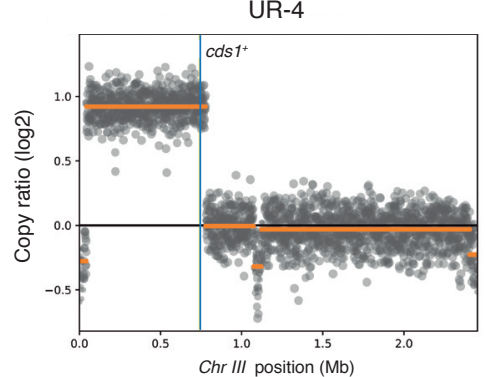

UR-14

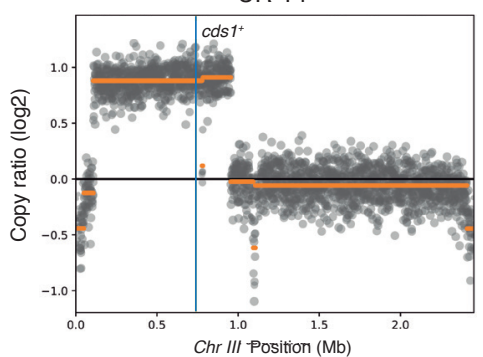

UR-19

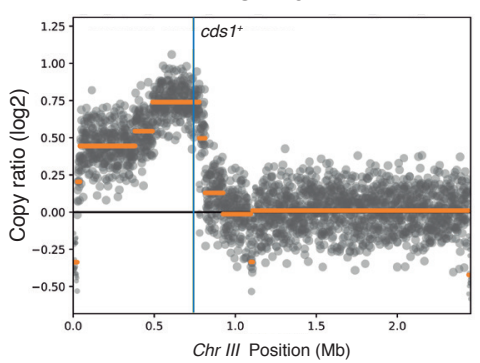

UR-5

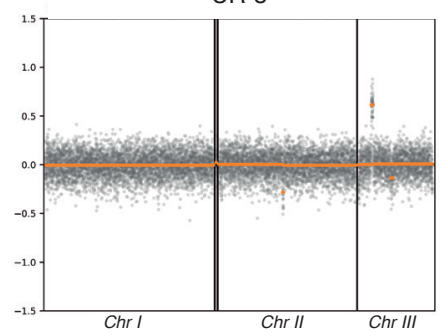

UR-15
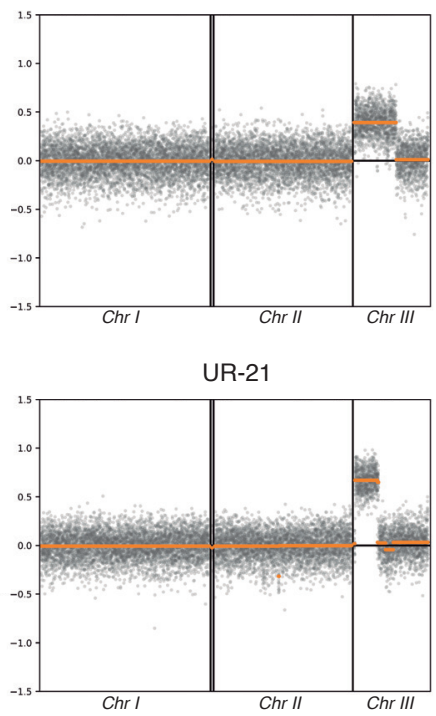

UR-5

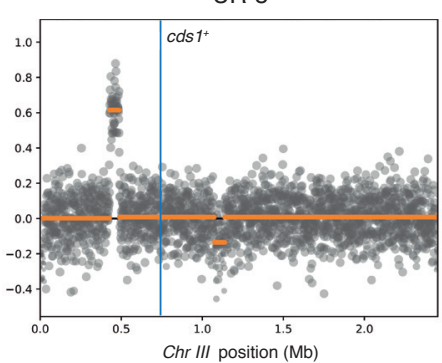

UR-15

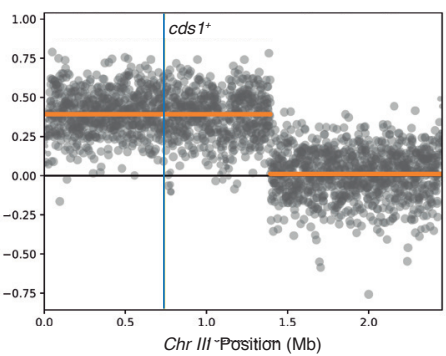

UR-21

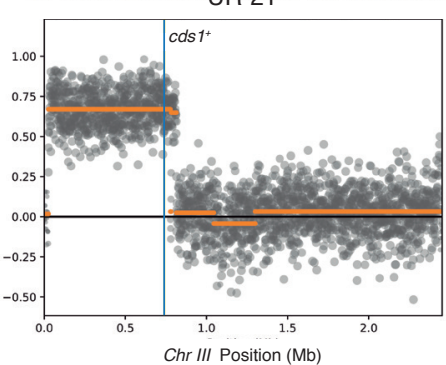

UR-6

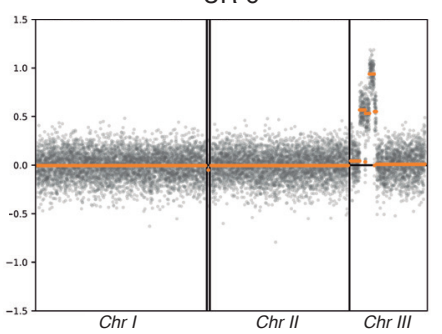

UR-17

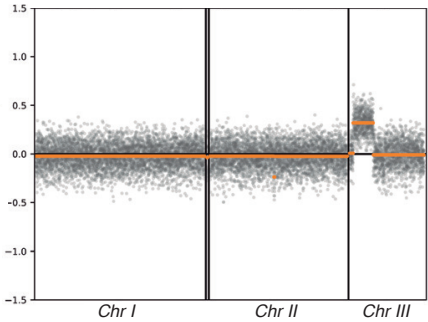

UR-22

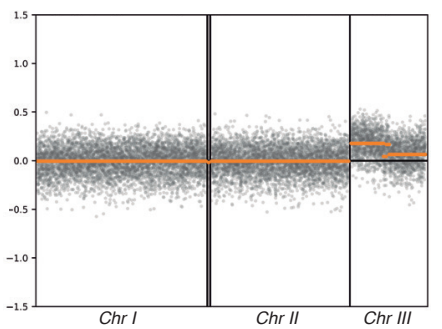

UR-6

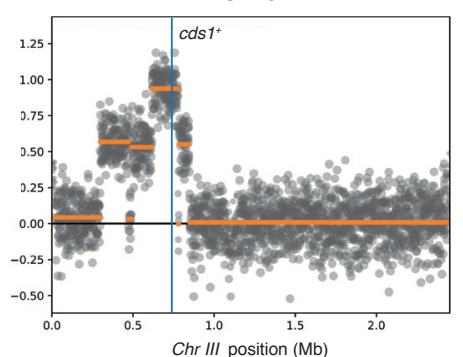

UR-17

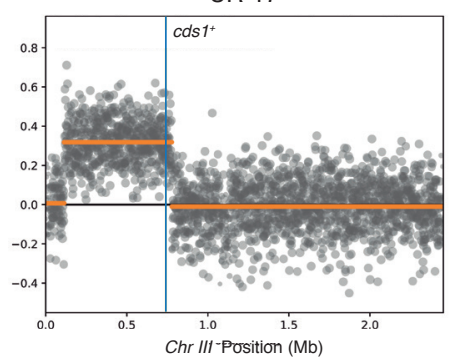

UR-22

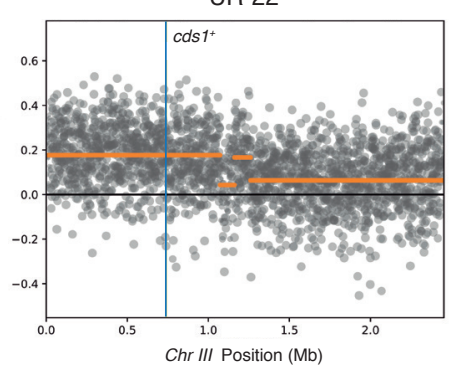

UR-13
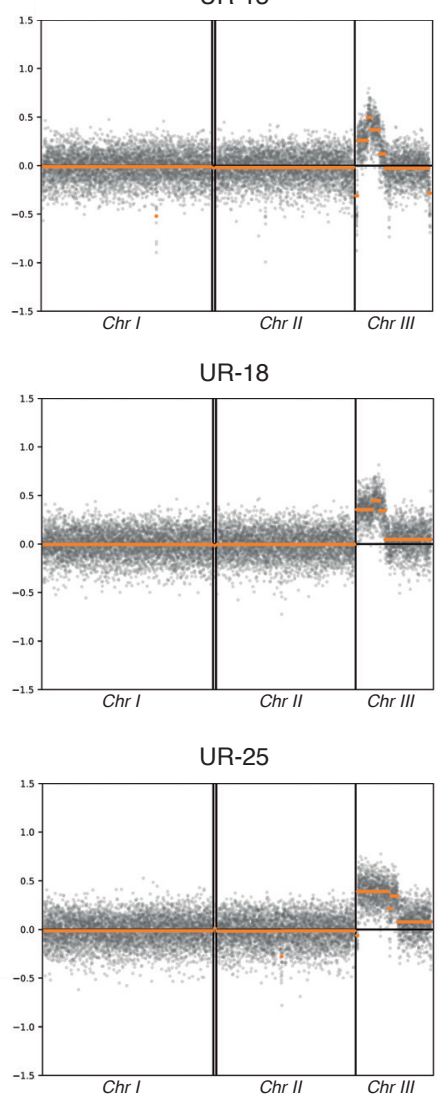

UR-13

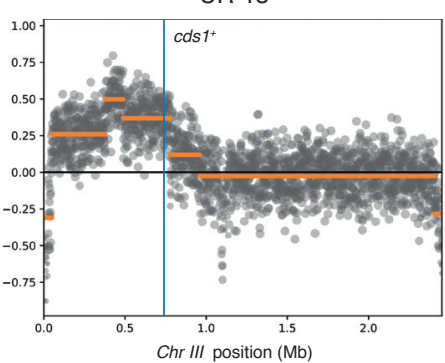

UR-18
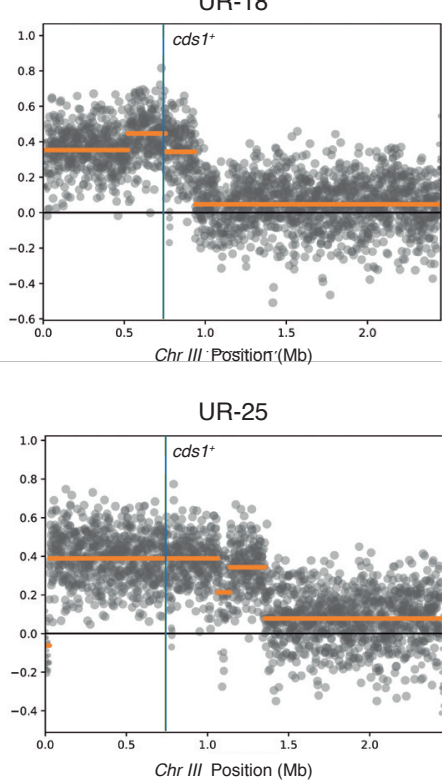

Extended Data Figure 6. Copy Number Variation (CNV) analysis reveals a partial duplication of chromosome III in 12 of 30 unstable (UR) caffeine-resistant isolates

a, Genome-wide coverage plots with overlaid segments in UR isolates showing partial duplication of chromosome III. Wild-type ChIP-seq input data were used as the reference.

b, Chromosome III coverage plots with overlaid segments in UR isolates showing partial duplication of chromosome III. Location of cds $1^{+}$is highlighted. Wild-type ChIP-seq input data were used as the reference. 


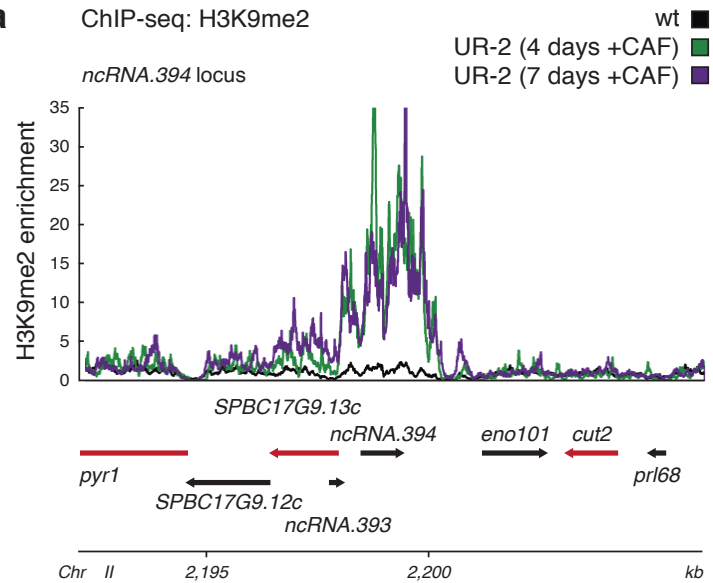

Whole Genome Sequencing

CNV analysis
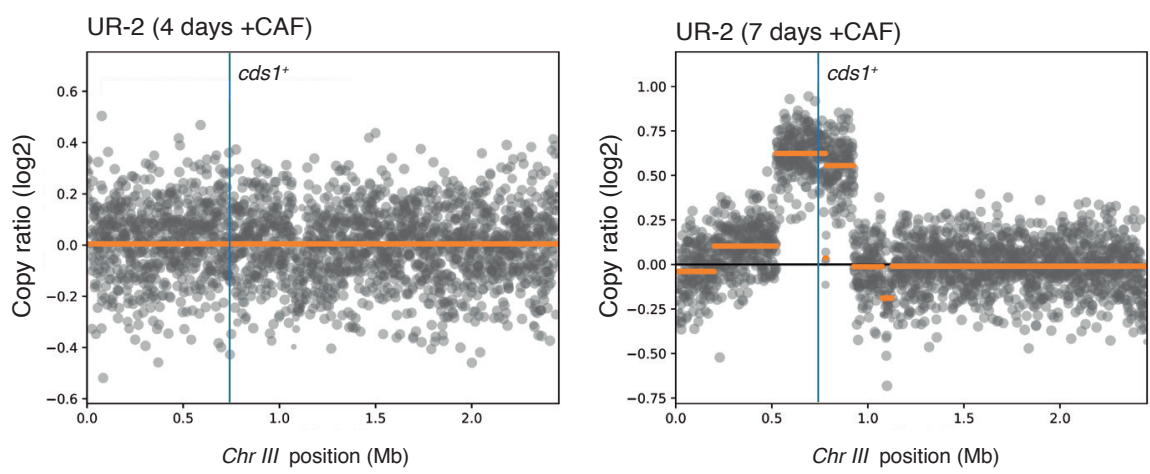

b

- CAF

wt

UR-2 (4 days $+\mathrm{CAF})$

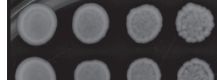
0000

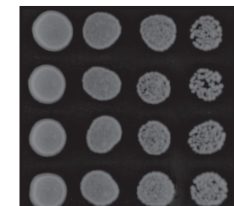

UR-2

(4 days $+\mathrm{CAF}$ )

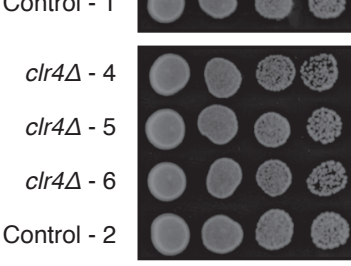

$+\mathrm{CAF}$
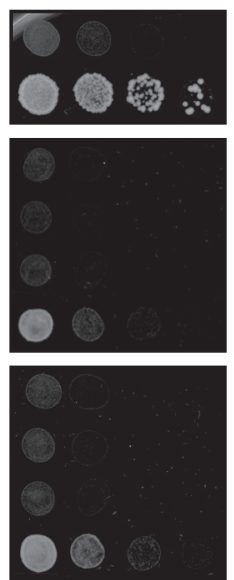

-CAF

wt

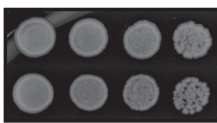

clr4 $\Delta-1$

clr4 4 - 2

clr4 $\Delta-3$

UR-2 ( 7 days $+\mathrm{CAF}$ )

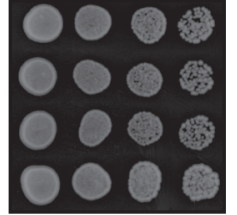

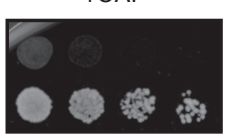

Whole Genome Sequencing

$\mathrm{CNV}$ analysis

ChIP-seq: H3K9me2 UR-2 ( 7 days + CAF) ncRNA.394 locus UR-2 ( 7 days + CAF $\rightarrow 14$ days - CAF $)$

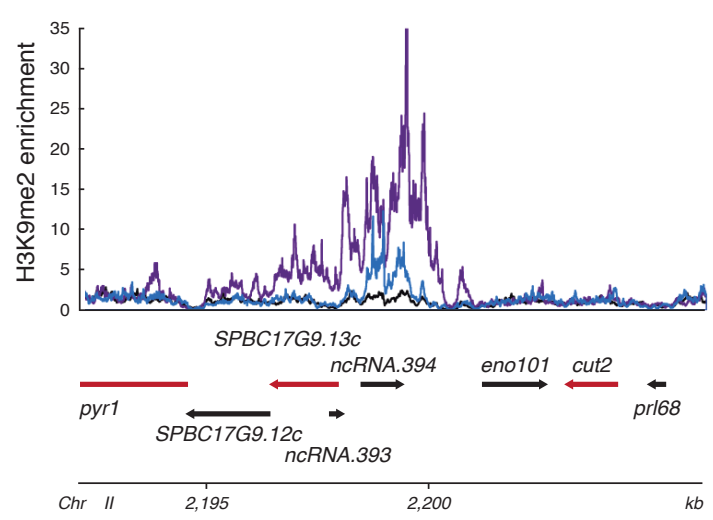

UR-2 (7 days +CAF)

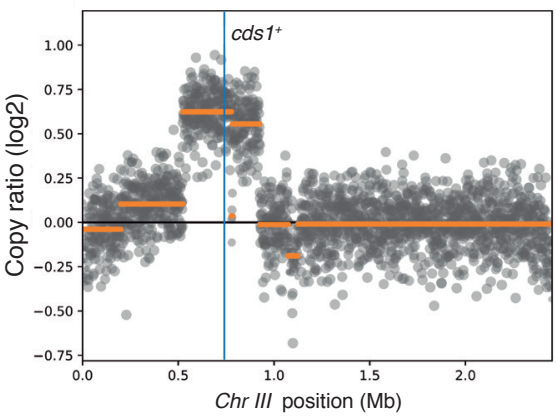

UR-2 $(7$ days $+\mathrm{CAF} \rightarrow 14$ days $-\mathrm{CAF})$

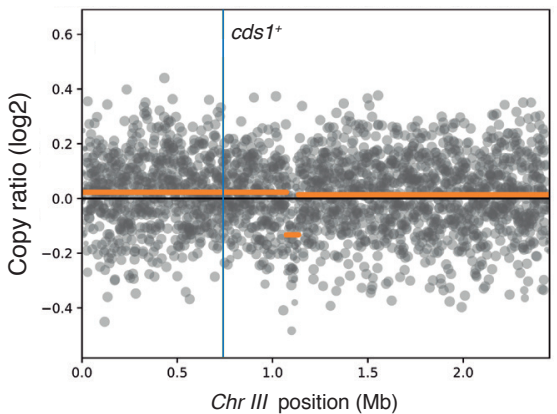

\section{Extended Data Figure 7. Epigenetic changes preceded genetic changes (CNV) in unstable caffeine-resistant isolate UR-2}

a, H3K9me2 ChIP-seq enrichment at the ncRNA.394 locus (left) and chromosome III coverage plots with overlaid segments (right) in UR-2 cells following prolonged growth on $+\mathrm{CAF}$ media for 3 days (7 days $+\mathrm{CAF}$ ). Wild-type ChIP-seq input data were used as the reference for CNV analysis.

b, $c / r 4^{+}(c / r 4 \Delta)$ or an unlinked intergenic region (Control) were deleted in UR-2 cells (4 days $+\mathrm{CAF}$ ) and UR-2 cells after prolonged growth on + CAF media for 3 days (7 days +CAF). All (6/6) UR-2 (4 days +CAF) clr4 $\Delta$ transformants lost resistance to caffeine whereas only $50 \%$ (3/6) UR-2 (7 days +CAF) lost resistance to caffeine.

c, H3K9me2 ChIP-seq enrichment at the ncRNA.394 locus (left) and chromosome III coverage plots with overlaid segments (right) in UR-2 cells following prolonged growth on non-selective media for 14 days after prolonged growth on + CAF media for 3 days (7 days + CAF --> 14 days -CAF). Wild-type ChIP-seq input data were used as the reference for CNV analysis. 

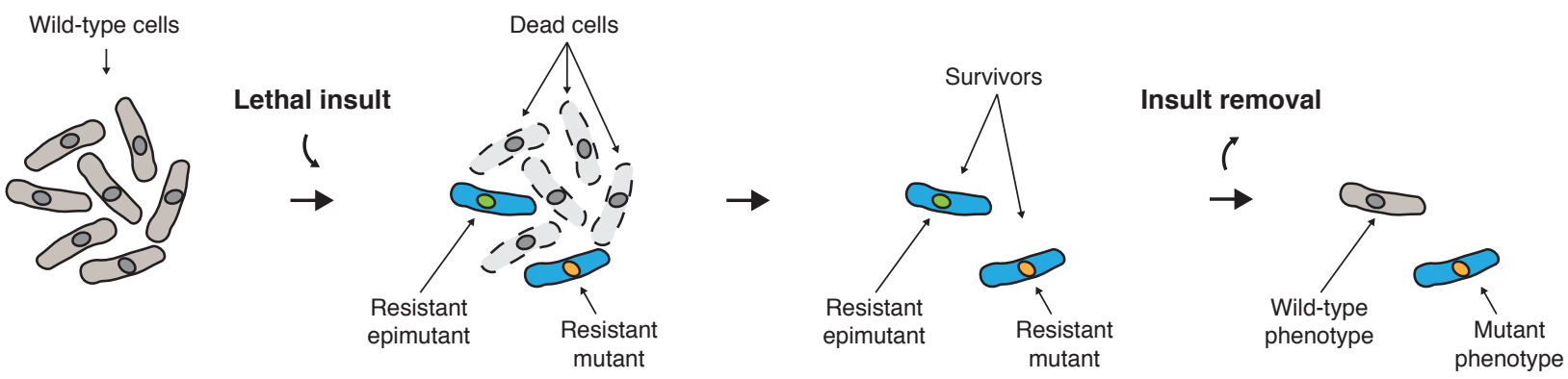

\section{Extended Data Figure 8. Model}

Resistant isolates arise following exposure to a lethal insult. Resistance might be mediated by permanent, DNA-based mutations (resistant mutants) or reversible, heterochromatin-based epimutations (resistant epimutants). Upon insult removal, resistant epimutants can revert to the wild-type phenotype by disassembling ectopic domains of heterochromatin, whereas resistant mutants continue displaying the mutant phenotype due to the genetic nature of DNA mutations. 